\title{
Picky predicates: why believe doesn't like interrogative complements, and other puzzles
}

\author{
Nadine Theiler ${ }^{1} \mathbb{C} \cdot$ Floris Roelofsen $^{1} \cdot$ Maria Aloni $^{1}$ \\ Published online: 18 March 2019 \\ (c) The Author(s) 2019
}

\begin{abstract}
It is a long-standing puzzle why predicates like believe embed declarative but not interrogative complements (e.g., Bill believes that/*whether Mary left) and why predicates like wonder embed interrogative but not declarative complements (e.g., Bill wonders whether/*that Mary left). This paper shows how the selectional restrictions of a range of predicates (neg-raising predicates like believe, truth-evaluating predicates like be true, inquisitive predicates like wonder, and predicates of dependency like depend on) can be derived from semantic assumptions that can be independently motivated.
\end{abstract}

Keywords Clause-embedding predicates $\cdot$ Selectional restrictions $\cdot$ Neg-raising

\section{Introduction}

Certain clause-embedding predicates take both declarative and interrogative complements, as shown in (1) for know. Others take only declarative complements, as illustrated in (2) for believe, or only interrogative complements, as seen in (3) for wonder.

(1) Bill knows that/whether/what Mary has eaten.

(2) Bill believes that/*whether/*what Mary has eaten.

(3) Bill wonders whether/what/*that Mary has eaten.

\begin{abstract}
We wish to thank two anonymous reviewers and our editor, Angelika Kratzer. Moreover, we are very grateful to Ivano Ciardelli, Michael Cohen, Alexandre Cremers, Donka Farkas, Clemens Mayr, Wataru Uegaki, and Yimei Xiang, as well as audiences at the University of Amsterdam, the University of Konstanz, and SALT for feedback on earlier incarnations of the ideas presented here. We also gratefully acknowledge funding from the European Research Council (ERC, Grant Number 680220) and the Netherlands Organisation for Scientific Research (NWO).
\end{abstract}

$\bowtie$ Nadine Theiler

nadine.theiler.87@gmail.com

1 ILLC, University of Amsterdam, Science Park 107, 1098 XG Amsterdam, The Netherlands 
Verbs like know are referred to as responsive predicates, predicates like wonder as rogative predicates, and predicates like believe as anti-rogative predicates. Any account that aims at explaining the distribution of clausal complements will have to capture both the selectional restrictions of rogative and anti-rogative predicates and the selectional flexibility of responsive predicates. Most accounts of clausal complements assume a type distinction between declarative and interrogative complements (e.g. Karttunen 1977; Heim 1994; Dayal 1996; Lahiri 2002; Spector and Egré 2015; Uegaki 2015b). Usually, declarative complements are taken to have type $\langle s, t\rangle$, while interrogative complements are taken to have type $\langle\langle s, t\rangle, t\rangle$. The selectional restrictions of (anti-) rogative predicates can then be captured by postulating that rogative predicates take arguments of type $\langle\langle s, t\rangle, t\rangle$, while anti-rogative predicates take arguments of type $\langle s, t\rangle$. On the other hand, to capture the selectional flexibility of responsive predicates, these accounts assume an operator that shifts the type of interrogatives into that of declaratives, or vice versa.

This approach, however, has its limitations. First, as soon as we admit type-shifting, we lose part of the account of selectional restrictions. This is because if we introduce an operator that adapts the type of interrogatives to that of declaratives (as in, e.g., Heim 1994), then this operator would also resolve the type conflict when antirogative predicates like believe take interrogative complements. Thus, in this case, we lose the account of the selectional restrictions of anti-rogatives. On the other hand, for analogous reasons, if the type-shifter adapts the type of declaratives to that of interrogatives (as in Uegaki 2015b), the account of the selectional restrictions of rogative predicates is lost. Thus, type-distinction-based accounts do not directly capture the selectional restrictions of both rogative and anti-rogative predicates at once. The selectional restrictions of one of these predicate classes need to be derived from factors other than the postulated type distinction between declaratives and interrogatives. Of course one may attempt to overcome this limitation by assuming that the type-shifter can only apply in certain configurations. The point here is that, without such additional assumptions, type-distinction-based accounts cannot capture selectional restrictions and selectional flexibility at the same time.

Moreover, if one wants to account for the selectional restrictions of rogative or anti-rogative predicates in terms of a type mismatch, one has to assume differences in semantic type between certain predicates which seem difficult to motivate independently. For instance, if one wants to account in this way for the fact that rogative predicates do not accept declarative complements while responsive predicates do, one has to assume a difference in semantic type between be curious (which is rogative; e.g., I'm curious who left / *that Bill left) and be of interest (which is responsive; e.g., Who left / that Bill left is of interest to me). Similarly, if one wants to explain the fact that anti-rogatives, unlike responsives, do not take interrogative complements in terms of a type mismatch, then one has to assume a difference in semantic type between predicates like assert and claim (which are anti-rogative) and ones like announce and state (which are responsive). In the absence of independent motivation for such type distinctions, the approach is stipulative to a certain degree. ${ }^{1}$ An account which derives

\footnotetext{
${ }^{1}$ It must be noted that such motivation is not completely absent: Uegaki (2015a) provides an explicit argument for his assumption that predicates like believe require an argument of type $\langle s, t\rangle$ while predicates
} 
the selectional restrictions of (anti-)rogatives from semantic assumptions about these predicates which can be independently motivated would be preferable.

The present paper assumes a uniform account of clausal complements, introduced in Theiler et al. 2018. The account is uniform in the sense that it assigns the same semantic type to declarative and interrogative complements, namely $\langle\langle s, t\rangle, t\rangle$, and assumes that all clause-embedding predicates take arguments of this type. On such an account, the selectional flexibility of responsive predicates is directly predicted, without any type-shifting operations. On the other hand, the selectional restrictions of (anti-)rogatives need to be explained based on independently observable properties of the relevant predicates. Such an explanation has recently been given for wonder and some closely related rogative predicates (Ciardelli and Roelofsen 2015; Uegaki $2015 b) .^{2}$ The present paper does so for another class of rogative predicates, namely predicates of dependency like depend on and be determined by, as well as two classes of anti-rogative predicates, namely (i) neg-raising predicates like believe and think and (ii) truth-evaluating predicates like be true and be false. Independently of the present paper, Mayr (2017) and Cohen (2017a, b) have also recently proposed ways to explain the anti-rogativity of neg-raising predicates. ${ }^{3}$ These accounts, while building on the same idea as ours, are more limited in scope and less explicit. We will discuss them in Appendix A.

The paper is structured as follows. Section 2 briefly lays out our uniform account of clausal complements, and exemplifies our treatment of responsive predicates. Section 3 is concerned with the selectional restrictions of anti-rogative predicates, Sect. 4 with those of rogative predicates, and Sect. 5 discusses an empirical and more general methodological issue. Section 6 concludes. Appendix A discusses related work in some detail, Appendix B spells out some technical details of the proposed account, and Appendix $\mathrm{C}$ presents an extension of the core account to presuppositional complements.

\section{A uniform treatment of clausal complements}

Our treatment of clausal complements is couched in inquisitive semantics (Ciardelli et al. 2013, 2015). ${ }^{4}$ In this framework, declarative and interrogative clauses are taken to have the same kind of semantic value, namely a set of propositions. The conceptual motivation behind this uniform notion of sentence meaning is as follows. While traditionally the meaning of a sentence $\varphi$ is taken to capture just the information conveyed

\footnotetext{
Footnote 1 continued

like know require an argument of type $\langle\langle s, t\rangle, t\rangle$. However, this argument does not seem entirely conclusive; see Appendix A.2 for discussion.

2 The proposals of Ciardelli and Roelofsen (2015) and Uegaki (2015b) are very much in the same spirit. For discussion of the subtle differences between them, see Appendix A.2.

3 A first version of the present account started circulating in the spring of 2016.

4 As will become clear in the course of the paper, this choice of framework is an integral part of the proposed account. In particular, as will be laid out in Sect. 3.1.3, in deriving the selectional restrictions of neg-raising predicates, we make crucial use of inquisitive negation as well as the fact that sentence meanings in inquisitive semantics are not arbitrary sets of propositions, but always downward closed.
} 
by $\varphi$, in inquisitive semantics it is taken to additionally capture the issue expressed by $\varphi$ as well. We call the information that is conveyed by a sentence its informative content, and the issue expressed by it its inquisitive content. To encode both kinds of content at once, the meaning of a sentence is construed as a set of propositions, no matter whether the sentence is declarative or interrogative.

By uttering a sentence $\varphi$ with meaning $\llbracket \varphi \rrbracket$, a speaker is taken to do two things at the same time. Firstly, she is taken to raise an issue whose resolution requires establishing one of the propositions in $\llbracket \varphi \rrbracket$. These propositions are called resolutions. Secondly, she is taken to provide the information that the actual world is contained in the union of all resolutions, $\bigcup \llbracket \varphi \rrbracket . \bigcup \llbracket \varphi \rrbracket$ is the informative content of $\varphi$, written as $\operatorname{info}(\varphi)$.

\subsection{Downward closure, alternatives, and truth}

Sentence meanings in inquisitive semantics are downward closed: if $p \in \llbracket \varphi \rrbracket$ and $q \subset p$, then also $q \in \llbracket \varphi \rrbracket$. This captures the intuition that, if a proposition $p$ resolves a given issue, then any stronger proposition $q \subset p$ will also resolve that issue. As a limit case, it is assumed that the inconsistent proposition, $\varnothing$, trivially resolves all issues, and is therefore included in the meaning of every sentence. The maximal elements in $\llbracket \varphi \rrbracket$ are referred to as the alternatives in $\llbracket \varphi \rrbracket$ and the set of these alternatives is denoted as $\operatorname{alt}(\varphi)$. Alternatives are those propositions that contain precisely enough information to resolve the issue expressed by $\varphi$. Finally, from the meaning of a sentence in inquisitive semantics, its truth conditions are derived in the following way: $\varphi$ is true in a world $w$ just in case $w$ is compatible with info $(\varphi)$, i.e., $w \in \operatorname{info}(\varphi)$.

For example, consider the sentence meaning $\llbracket \varphi \rrbracket=\left\{\left\{w_{1}, w_{2}\right\},\left\{w_{1}\right\},\left\{w_{2}\right\}, \varnothing\right\}$. This meaning contains exactly four resolutions, namely $\left\{w_{1}, w_{2}\right\},\left\{w_{1}\right\},\left\{w_{2}\right\}$, and $\varnothing$. It contains exactly one alternative, namely $\left\{w_{1}, w_{2}\right\}$. That is, $\operatorname{alt}(\varphi)=\left\{\left\{w_{1}, w_{2}\right\}\right\}$. Since $\llbracket \varphi \rrbracket$ is downward closed, it additionally contains all subsets of $\left\{w_{1}, w_{2}\right\}$, i.e., $\left\{w_{1}\right\},\left\{w_{2}\right\}$, and $\varnothing$. The informative content of $\varphi$ is info $(\varphi)=\left\{w_{1}, w_{2}\right\}$. This means that $\varphi$ is true in $w_{1}$ and $w_{2}$ and false in all other worlds.

\subsection{Informative and inquisitive sentences}

The informative content of $\varphi$ can be trivial, namely iff the propositions in $\llbracket \varphi \rrbracket$ cover the entire logical space $W$, i.e., iff info $(\varphi)=W$. In this case, we call $\varphi$ non-informative. Conversely, we call $\varphi$ informative iff info $(\varphi) \neq W$. Not only the informative content, but also the inquisitive content of a sentence can be trivial. This is the case iff the issue expressed by $\varphi$ is already resolved by the information provided by $\varphi$ itself, i.e., iff $\operatorname{info}(\varphi) \in \llbracket \varphi \rrbracket$. In this case, we call $\varphi$ non-inquisitive. Conversely, $\varphi$ is called inquisitive iff $\operatorname{info}(\varphi) \notin \llbracket \varphi \rrbracket$. If $\varphi$ is non-inquisitive, its meaning contains a unique alternative, namely info $(\varphi)$. Vice versa, if $\llbracket \varphi \rrbracket$ contains multiple alternatives, it is inquisitive. 

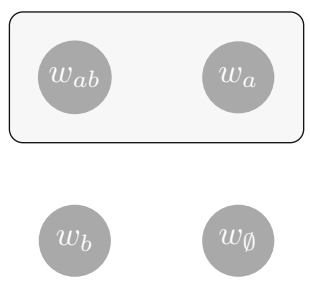

(a) that Ann left
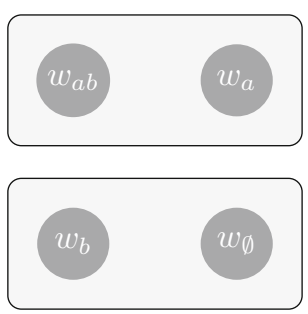

(b) whether Ann left

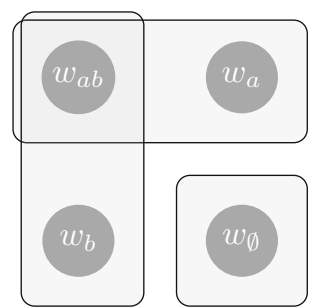

(c) who left

Fig. 1 Examples of complement clause meanings in inquisitive semantics

\subsection{Declarative and interrogative complements}

Following Ciardelli et al. (2015) and much earlier work in inquisitive semantics, we assume that a declarative complement or matrix clause $\varphi$ is never inquisitive. ${ }^{5}$ That is, its meaning $\llbracket \varphi \rrbracket$ always contains a single alternative, which coincides with its informative content, info $(\varphi)$. For example:

$$
\text { alt(that Ann left })=\{\{w \mid \text { Ann left in } w\}\}
$$

Conversely, we assume that an interrogative complement or matrix clause is never informative. This means that the alternatives associated with an interrogative clause always completely cover the set of all possible worlds. ${ }^{6}$ For example, if the domain of discourse consists of Ann and Bob, we assume the following sets of alternatives for the interrogative complements whether Ann left and who left. ${ }^{7}$

$$
\begin{aligned}
& \text { alt(whether Ann left })=\left\{\begin{array}{l}
\{w \mid \text { Ann left in } w\}, \\
\{w \mid \text { Ann didn't leave in } w\}
\end{array}\right\} \\
& \text { alt(who left })=\left\{\begin{array}{l}
\{w \mid \text { Ann left in } w\}, \\
\{w \mid \text { Bob left in } w\}, \\
\{w \mid \text { nobody left in } w\}
\end{array}\right\}
\end{aligned}
$$

The alternative sets in (4)-(6) are also depicted in Fig. 1, where $w_{a b}$ is a world in which both Ann and Bill left, $w_{a}$ one in which only Ann left, $w_{b}$ one in which only Bill left, and $w_{\emptyset}$ one in which neither Ann nor Bill left.

\footnotetext{
5 There is also work in inquisitive semantics that does not make this assumption (e.g. AnderBois 2012). This requires a view under which uttering an inquisitive sentence does not necessarily involve issuing a request for information. See Ciardelli et al. (2012) for discussion.

${ }^{6}$ For simplicity we leave the presuppositions of complement clauses out of consideration here; Appendix C discusses how the proposed account can be extended to deal with such presuppositions.

7 The alternatives assumed here for $w h$-interrogatives only allow us to derive non-exhaustive (mentionsome) readings. Our account can be refined to derive intermediate and strongly exhaustive readings as well (see Theiler et al. 2018). This refinement doesn't affect any of the results presented here.
} 


\subsection{Responsive predicates: a brief illustration}

Before dealing with the selectional restrictions of anti-rogative predicates, let us first briefly specify a basic lexical entry for the responsive predicate be certain, showing that its selectional flexibility is immediately captured. ${ }^{8}$ In the entry below, $P$ is the meaning of the clausal complement, its semantic type $\langle\langle s, t\rangle, t\rangle$ is abbreviated as $T$, and $\operatorname{DOX}_{x}^{w}$ is the doxastic state of the subject $x$ in world $w .{ }^{9}$

$$
\llbracket \text { be certain } \rrbracket^{w}=\lambda P_{T} \cdot \lambda x . \mathrm{DOX}_{x}^{w} \in P
$$

As illustrated by the following examples, this entry uniformly handles declarative and interrogative complements, which are both of type $T$.

Mary is certain that John left.

$\rightsquigarrow$ True in $w$ iff $\operatorname{DOX}_{m}^{w} \subseteq\{w \mid$ John left in $w\}$

(9) Mary is certain who left.

$\rightsquigarrow$ True in $w$ iff $\exists p \in\left\{\begin{array}{l|l}\{w \mid & \text { Ann left in } w\}, \\ \{w \mid & \text { Bob left in } w\}, \\ \{w \mid & \text { nobody left in } w\}\end{array}\right\}$ s.t. $\operatorname{DOX}_{m}^{w} \subseteq p$

The present approach thus yields a more economical treatment of responsive predicates than approaches that assume a type distinction between declarative and interrogative complements. It is not necessary here to assume a type-shifting operation (or multiple lexical entries for each responsive predicate). Moreover, as discussed in Theiler et al. 2018, the approach avoids certain thorny problems, brought to light in George 2011, Elliott et al. 2017, Roberts 2018, and Uegaki and Roelofsen 2018, for mainstream theories which assume a type-shifting operation from sets of propositions to propositions. It should be noted, however, that these problems are also avoided by the approach of Uegaki (2015b), which assumes a type-shifting operation in the opposite direction. ${ }^{10}$

\footnotetext{
${ }^{8}$ For a full account of be certain this basic entry needs to be refined. For instance, the given entry does not capture the fact that when taking a wh-complement be certain only permits a strongly exhaustive reading (Uegaki 2015b; Theiler et al. 2018), nor the fact that when taking a polar interrogative complement, the predicate is degraded in plain episodic, positive sentences, while completely fine under negation (Mayr 2017; van Gessel et al. 2018). These empirical facts are left out of consideration here.

${ }^{9}$ For simplicity, we give truth-conditional entries here. For a full-fledged compositional inquisitive semantics, these can easily be transformed into support-conditional entries; see Appendix B.

10 A general argument that has been made against the uniform approach taken here is that it does not impose any constraints on the space of possible responsive predicate meanings (George 2011; Spector and Egré 2015). In defense of the approach, Theiler et al. (2018) point out that the same situation exists in other empirical domains, and that the solutions proposed there may be applied in the domain of responsive predicates as well. For instance, generalized quantifier theory leaves the space of possible determiner meanings highly unconstrained. Only a small subset of these meanings can be expressed by lexical determiners in natural languages. It has been argued that this may be rooted in the fact that certain types of determiner meanings are significantly harder to learn and/or process than others (see, e.g., Steinert-Threlkeld and Szymanik 2019). Steinert-Threlkeld (2019) and Theiler et al. (2018) suggest that the same approach may be taken in the domain of responsive predicates, and take some concrete steps in this direction.
} 


\section{Anti-rogative predicates}

We will now turn our attention to anti-rogative predicates, which include attitude predicates like think and believe, ${ }^{11}$ likelihood predicates such as seem and be likely, speech-act predicates like claim and assert, truth-evaluating predicates like be true and be false, and non-veridical preferential predicates like hope and fear. We won't account for the anti-rogativity of all these different predicate classes here, but instead will focus on just two classes, namely neg-raising predicates such as believe, think, seem, and be likely (Sect. 3.1) and the truth-evaluating predicates be true and be false (Sect. 3.2).

\subsection{Neg-raising predicates}

\subsubsection{Zuber's observation: all neg-raising predicates are anti-rogative}

It has been observed that-diverse as the class of anti-rogative predicates may bethere is something that many of them have in common: namely, many of them are negraising. This means, at first pass, that they license the following kind of inference: ${ }^{12}$

Mary does not believe that Ann left.

$\therefore$ Mary believes that Ann did not leave.

Zuber (1982) claims that all neg-raising predicates are anti-rogative. Indeed, examining the class of neg-raisers, it doesn't seem possible to find a counterexample to this generalization. Some anti-rogative neg-raisers are given in (11).

believe, think, feel, expect, want, seem, be likely

${ }_{11}$ In this paper, we will set aside the observation that in certain constructions believe does in fact take interrogative complements. Two examples are given in (i.a-b):

(i) a. You won't believe who won!

b. He just wouldn't believe me who I was.

c. *You won't think who won!

d. *You won't believe whether Mary won!

e. *You won't believe who called in ages!

Note that, as illustrated in (i.c), other anti-rogative predicates do not seem to exceptionally license interrogative complements in these configurations, and as illustrated in (i.d), while believe exceptionally licenses wh-interrogatives in these cases, polar interrogative complements are still unacceptable. Interestingly, when believe felicitously embeds an interrogative complement, it becomes factive. This means that believe in these constructions shares many properties with emotive factive predicates like be surprised. Finally, it was pointed out by an anonymous reviewer that believe in these configurations is not neg-raising. This can be observed from the ungrammaticality of (i.e): if believe was neg-raising, it would license the strong NPI in ages (Gajewski 2007). Since we will derive the anti-rogativity of believe from the fact that it is neg-raising, this last observation might be taken to corroborate our account. Further investigation of this peculiar construction must be left for another occasion, though.

12 See, e.g., Horn (1989) and Gajewski (2007) for a characterization of neg-raising predicates in terms of strict NPI licensing, which is arguably more reliable but would take us a bit far afield here. 
We will show that once we add a treatment of neg-raising to our present account of clausal embedding, then, indeed, anti-rogativity will follow. In our discussion we will focus on the case of believe, and indicate how the account can be extended to other neg-raising predicates.

Note, however, that Zuber's generalization does not hold in the other direction; there are several anti-rogative predicates that are not neg-raising:
a. Truth-evaluating predicates: be true, be false $e^{13}$
b. Non-veridical preferential predicates: e.g., desire, fear
c. Speech act predicates: e.g., claim, assert

This means that an analysis which derives anti-rogativity from neg-raising will not cover all anti-rogative predicates. As mentioned above, we will consider the truthevaluating predicates be true and be false in Sect. 3.2, and will briefly return to the anti-rogativity of the remaining predicates in (12) in Sect. 6.

\subsubsection{Deriving neg-raising from an excluded-middle presupposition}

We start with a preliminary entry for believe, which is identical to the basic entry for be certain given in Sect. 2.4 and which doesn't yet capture the fact that believe is neg-raising.

$$
\llbracket \text { believe } \rrbracket^{w}=\lambda P_{T} \cdot \lambda x . \operatorname{DOx}_{x}^{w} \in P
$$

(preliminary entry)

We adopt a presuppositional account of neg-raising, which was originally proposed by Bartsch (1973) and further developed by Gajewski (2007). ${ }^{14}$ On this account, neg-raising behavior results from a so-called excluded-middle (EM) presupposition, carried by all neg-raising predicates. For instance, sentence (14) presupposes that Mary is opinionated as to whether Ann left: she either believes that Ann left or she believes that Ann didn't leave.

Mary believes that Ann left.

Presupposition: M believes that A left or M believes that A didn't leave.

13 Be truelfalse aren't categorized as neg-raising here, although they do license neg-raising inferences. This is because, as illustrated in (i), negated be truelfalse don't license strict NPIs, unlike predicates like think and believe; see also footnote 12 above.
a. *It isn't true that Mary will leave until June.
b. John doesn't think that Mary will leave until June.

As we will discuss in a moment, we will assume that neg-raising predicates involve a so-called excludedmiddle presupposition (Bartsch 1973; Gajewski 2007). Assuming that be true/false involve such a presupposition would (i) make wrong predictions about the licensing of strict NPIs, and (ii) amount to assuming a tautological presupposition for these predicates (since it is true for any proposition $p$ that $p$ is true or that $\neg p$ is true).

14 Besides the presuppositional account of neg-raising, there are also accounts based on implicatures (e.g., Romoli 2013) or homogeneity (Gajewski 2005; Križ 2015); see Križ (2015, Ch.6) for a recent overview and comparison. We leave open at this point whether the generalization that neg-raising predicates are anti-rogative can also be derived on these other accounts. 
In (14), the presupposition easily goes unnoticed, though, since it is weaker than the asserted content. On the other hand, if we negate (14), presupposed and asserted content become logically independent. Taken together, they imply that Mary believes that Ann didn't leave-which accounts for the neg-raising effect.

Mary doesn't believe that Ann left.

Presupposition: $\mathrm{M}$ believes that A left or $\mathrm{M}$ believes that A didn't leave.

$\therefore$ Mary believes that Ann didn't leave.

It should be noted, as Bartsch does herself, that neg-raising is defeasible: if the opinionatedness assumption is suspended, as in (16), believe receives a non-neg-raising reading. This behavior sets neg-raising predicates apart from certain other presupposition triggers, such as it-clefts, whose presuppositions are hard to cancel or to locally accommodate under sentential negation.
Bill doesn't know who killed Caesar. He isn't even sure whether or not Brutus and Caesar lived at the same time. So, naturally...
Bill doesn't believe that Brutus killed Caesar.
$\leftrightarrow$ Bill believes that Brutus didn't kill Caesar.

One might think that the easy defeasibility of neg-raising makes it more attractive to treat the EM inference as a conversational implicature. This option, however, was convincingly rejected by Horn (1978), who argued that there is no obvious semantic property determining whether a predicate is neg-raising or not. For instance, while want is neg-raising, the closely related desire is not.

We therefore maintain a presuppositional account like that of Bartsch, and additionally assume, following Gajewski (2007), that the excluded-middle presupposition is locally accommodated in cases like (16) in order to obtain an interpretation that is consistent with the contextually given information. ${ }^{15}$

\subsubsection{A generalized EM presupposition}

If we want to add the EM presupposition to our uniform lexical entry for believe, repeated in (17), there is one more thing to take into account.

$$
\llbracket \text { believe } \rrbracket^{w}=\lambda P_{T} \cdot \lambda x . \operatorname{DOx}_{x}^{w} \in P
$$

(preliminary entry)

The semantic object $P$ that believe takes as its argument on our account is not a single proposition but a downward-closed set of propositions. If we compute its negation simply by taking its set-theoretical complement, this does not yield the desired result. ${ }^{16}$

\footnotetext{
15 Gajewski (2007) emphasizes that the excluded-middle presupposition of neg-raising predicates, because of its defeasibility, should be regarded as a soft presupposition in the sense of Abusch (2002, 2010). However, his actual account of their defeasibility is in terms of local accommodation and does not seem to explicitly rely on the assumption that they are soft presuppositions in Abusch's sense. It does assume, of course, that their local accommodation under negation is relatively unproblematic, in contrast with presuppositions contributed by other triggers, such as it-clefts.

16 To see this, consider again a model with $W=\left\{w_{a b}, w_{a}, w_{b}, w_{\emptyset}\right\}$. In this model, the meaning of the declarative complement that Ann left is 【that Ann left $\rrbracket=\left\{\left\{w_{a b}, w_{a}\right\},\left\{w_{a b}\right\},\left\{w_{a}\right\}, \varnothing\right\}$, as depicted in
} 

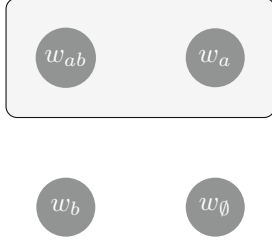

(a) that Ann left

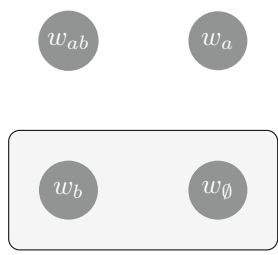

(b) that Ann didn't leave
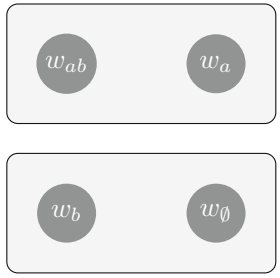

(c) whether Ann left

Fig. 2 Example illustrating inquisitive negation

We will therefore use the negation operation from inquisitive semantics, written as $\pi$. When applied to a sentence meaning $P$, inquisitive negation returns the set of those propositions that are inconsistent with every member of $P:{ }^{17}$

$$
\neg P:=\{p \mid \forall q \in P: q \cap p=\emptyset\}
$$

This operation may be thought of as a generalized negation that can be applied to both declarative and interrogative clauses. For declarative clauses, the result corresponds to what we would expect from a classical negation operation. For instance, take the sentence meaning $P=\llbracket$ that Ann left $\rrbracket=\left\{\left\{w_{a b}, w_{a}\right\},\left\{w_{a b}\right\},\left\{w_{a}\right\}, \varnothing\right\}$, depicted in Fig. 2a. Applying inquisitive negation to this sentence meaning yields:

$$
\begin{aligned}
\rightarrow P= & \{p \mid \forall q \in P: q \cap p=\emptyset\} \\
= & \left\{p \mid\left\{w_{a b}, w_{a}\right\} \cap p=\emptyset \text { and }\left\{w_{a b}\right\} \cap p=\emptyset \text { and }\left\{w_{a}\right\} \cap p=\emptyset\right. \\
& \quad \text { and } \emptyset \cap p=\emptyset\} \\
= & \left\{p \mid\left\{w_{a b}, w_{a}\right\} \cap p=\emptyset\right\} \\
= & \left\{\left\{w_{b}, w_{\emptyset}\right\},\left\{w_{b}\right\},\left\{w_{\emptyset}\right\}, \emptyset\right\}
\end{aligned}
$$

Observe that, as expected, this result corresponds to the meaning of that Ann didn't leave, depicted in Fig. 2b.

For interrogative clauses, applying inquisitive negation always yields the inconsistent sentence meaning, $\{\varnothing\}$. To see why, recall from Sect. 2 that interrogative clauses are never informative: the alternatives in the meaning of an interrogative complement, taken together, always cover the entire logical space. ${ }^{18}$ As a consequence, there can be

\section{Footnote 16 continued}

Fig. 2a. We expect of a suitable negation operation that, when applied to this sentence meaning, it yields

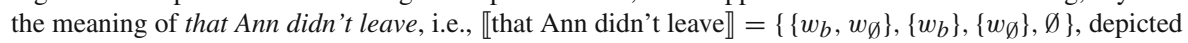
in Fig. 2b. However, if we implement negation as set-theoretic complementation, we don't get this result.

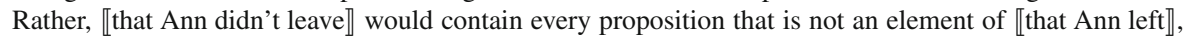
including, e.g., the trivial proposition $W$ and the proposition that Bob didn't leave, $\left\{w_{a}, w_{\emptyset}\right\}$.

17 There is both conceptual and empirical support for this way of treating negation in inquisitive semantics. Conceptually, it can be characterized in terms of exactly the same algebraic properties as the standard truthconditional negation operator (Roelofsen 2013a). Empirical support comes, for instance, from sluicing constructions (AnderBois 2014). Note also that an analogous treatment of negation has been proposed in alternative semantics (Kratzer and Shimoyama 2002).

18 Recall that we leave presuppositional complement clauses out of consideration here; see Appendix C for an extension of the core account developed here that deals with such complements. 
no non-empty proposition that is inconsistent with every proposition in an interrogative sentence meaning. For a concrete example, consider the interrogative sentence meaning $Q=\llbracket$ whether Ann left $\rrbracket=\left\{\left\{w_{a b}, w_{a}\right\},\left\{w_{a b}\right\},\left\{w_{a}\right\},\left\{w_{b}, w_{\emptyset}\right\},\left\{w_{b}\right\},\left\{w_{\emptyset}\right\}, \varnothing\right\}$, depicted in Fig. 2c. Applying inquisitive negation to $Q$ yields:

$$
\begin{aligned}
\rightarrow Q & =\{p \mid \forall q \in Q: q \cap p=\emptyset\} \\
& =\left\{p \mid\left\{w_{a b}, w_{a}\right\} \cap p=\emptyset \text { and }\left\{w_{a b}\right\} \cap p=\emptyset \text { and }\left\{w_{a}\right\} \cap p=\emptyset\right. \text { and } \\
& \left.\quad\left\{w_{b}, w_{\emptyset}\right\} \cap p=\emptyset \text { and }\left\{w_{b}\right\} \cap p=\emptyset \text { and }\left\{w_{\emptyset}\right\} \cap p=\emptyset \text { and } \emptyset \cap p=\emptyset\right\} \\
& =\left\{p \mid\left\{w_{a b}, w_{a}\right\} \cap p=\emptyset \text { and }\left\{w_{b}, w_{\emptyset}\right\} \cap p=\emptyset\right\} \\
& =\{\emptyset\}
\end{aligned}
$$

Using inquisitive negation, we can now formulate a lexical entry for believe including the EM presupposition. We will refer to the EM presupposition in this setting as the generalized EM presupposition, as it applies to both declarative and interrogative complements.

$$
\llbracket \text { believe } \rrbracket^{w}=\lambda P_{T} \cdot \lambda x: \underline{\operatorname{DOx}_{x}^{w} \in P \vee \operatorname{DOx}_{x}^{w} \in \pi^{P}} . \operatorname{DOx}_{x}^{w} \in P
$$

What will be crucial for our account of the selectional restrictions of neg-raising predicates is that the effect of the generalized EM presupposition depends on whether the complement is declarative or interrogative.

Declarative complements. As discussed in Sect. 2, we assume that declarative complements are never inquisitive. This means that if $P$ is the meaning of a declarative complement, it contains only one alternative $p$. Then, the first disjunct in the presupposition amounts to $\operatorname{DOX}_{x}^{w} \subseteq p(x$ believes that $p)$, while the second disjunct amounts to $\operatorname{DOX}_{x}^{w} \cap p=\varnothing(x$ believes that $\neg p)$. Hence, for declarative complements, our generalized rendering of the EM presupposition boils down to the ordinary version of this presupposition.

Interrogative complements. On the other hand, as discussed above, interrogative complements are never informative, as a result of which the inquisitive negation of an interrogative complement meaning $P$ is always $\rightarrow P=\{\emptyset\}$. Hence, the second disjunct of the presupposition can only be satisfied if $\operatorname{DOX}_{x}^{w}=\emptyset$. Under the standard assumption that doxastic states are consistent, this is impossible. In other words, the second disjunct is redundant.

However, what if we are not willing to make the assumption that doxastic states, or more generally, modal bases are consistent? After all, our eventual aim will be to account for the anti-rogativity of all neg-raising predicates, not only for that of believe, and different neg-raising predicates have different modal bases. Taking it for granted that all of these modal bases are necessarily consistent would be too strong an assumption. Fortunately, even without this assumption, the second disjunct in the presupposition turns out to be redundant. To see this, assume that the second disjunct is true, i.e., that $\operatorname{DOX}_{x}^{w}=\emptyset$. Then, the first disjunct amounts to the condition that $\emptyset \in P$. Since $P$ is an interrogative complement meaning, the propositions that it contains together cover the entire logical space. Therefore, since sentence meanings 
are downward closed, the condition that $\emptyset \in P$ is always satisfied. ${ }^{19}$ More generally, whenever the second disjunct is true, the first is true as well. Thus, the second disjunct in the presupposition is redundant, and this is the case with or without the assumption that doxastic states are consistent. As a consequence, if believe takes an interrogative complement, its lexical entry reduces to (20).

$$
\llbracket \text { believe } \rrbracket^{w}=\lambda P_{T} \cdot \lambda x: \underline{\operatorname{DOx}_{x}^{w} \in P} . \operatorname{DOx}_{x}^{w} \in P
$$

The presupposed and the asserted content in (20) are exactly the same. This means that when believe combines with an interrogative complement, its assertive component is trivial relative to its presupposition. Prima facie, we would expect triviality like this to make itself felt as logical deviance. But this is not what we find in this case: when believe combines with an interrogative complement, we perceive the result as ungrammatical. Can we explain ungrammaticality in terms of logical deviance? Gajewski (2002) argues that this is indeed possible for certain cases of systematic triviality. In the following sections we will show that the triviality observed above is indeed a case of such systematic triviality, and we will spell out in detail how Gajewski's theory can be applied to explain the anti-rogative nature of neg-raising predicates.

\subsubsection{L-analyticity}

What we mean here by systematic triviality is that the meaning of a sentence in which a neg-raising predicate embeds an interrogative complement comes out as trivial independently of the exact lexical material that appears in the sentence. In particular, it doesn't matter which exact predicate is used - the triviality only depends on the fact that the predicate is neg-raising — and it doesn't matter which lexical material appears in the complement-the triviality only depends on the fact that the complement is interrogative.

In contrast, there are also cases of non-systematic triviality such as the tautology in (21), which does rely on the presence of specific lexical material.

Every tree is a tree.

Gajewski (2002) suggests that cases of systematic triviality can be delineated from cases of non-systematic triviality in terms of the notion of logical analyticity (for short, L-analyticity). If a sentence is L-analytical, we do not perceive its triviality as logical deviance, as we do in cases of non-systematic triviality such as (21). Rather, according to Gajewski, L-analyticity manifests itself at the level of grammar: L-analytical sentences are perceived as being ungrammatical. An example of a phenomenon that Gajewski accounts for using this line of argument is the definiteness restriction in existential statements, exemplified in (22). Below we will see how he recasts a prominent analysis of this restriction, originally due to Barwise and Cooper (1981), in terms of L-analyticity.

\footnotetext{
19 Note that at this point one particular feature of inquisitive semantics, namely the downward-closedness of sentence meanings, is crucial for the proposed account.
} 
(22) *There is every tall tree.

Logical words. The notion of L-analyticity builds upon the distinction between logical and non-logical vocabulary. Intuitively, this distinction is easy to grasp; it runs along the lines of words that have lexical content versus words that don't. Among the logical words are quantifiers like $a$ or every, connectives like and or if, and copulas like is. Among the non-logical words, on the other hand, are predicates like tree, run, and green. There is no general agreement in the literature on a single definition of the class of logical words. Abrusán (2014) provides an overview of definitions that have been proposed, most of them based on invariance conditions. For the purposes of this paper, we will assume that a suitable definition of logical words can in principle be given. As far as we can see, the items that we will classify as logical are uncontroversially so, meaning that they should come out as logical under any suitable definition of logicality.

Logical skeleton. To determine whether a given sentence is L-analytical, we first compute its logical skeleton (LS) using the algorithm from Gajewski (2002). Let $\alpha$ be the logical form (LF) of the sentence. Then we obtain the LS from $\alpha$ by (i) identifying the maximal constituents of $\alpha$ that don't contain any logical items, and (ii) replacing each such constituent $\beta$ with a fresh constant of the same type as that of $\beta$. For example, the LFs and LSs of Every tree is a tree and There is every tall tree are given in (23) and (24). In (23), the maximal constituents of the LF not containing any logical items are the two instances of tree. In (24), the only maximal non-logical constituent of the LF is the phrase tall tree.

(23) Every tree is a tree.

\section{Logical form:}

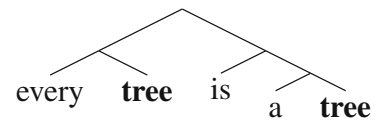

Logical skeleton:
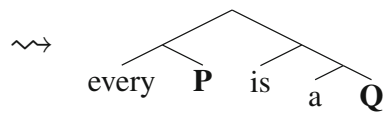

*There is every tall tree.

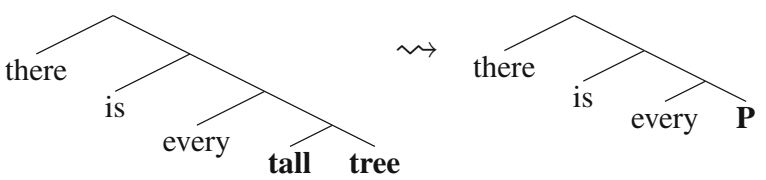

L-analyticity and ungrammaticality. We adopt the following assumptions about L-analyticity and ungrammaticality from Gajewski (2009).

Assumption 1 (L-analyticity) A sentence $S$ is L-analytical just in case $S$ 's LS receives the denotation 1 (or 0 ) for all interpretations in which its denotation is defined.

Assumption 2 (Ungrammaticality) A sentence is ungrammatical if it contains an L-analytical constituent.

For example, consider the interpretation of the LS in (23): 


$$
\llbracket \text { every } \mathbf{P} \text { is a } \mathbf{Q} \rrbracket^{\langle D, I\rangle}=\llbracket \text { every } \rrbracket^{\langle D, I\rangle}(I(\mathbf{P}))(I(\mathbf{Q}))
$$

It is possible to find two interpretations $I_{1}$ and $I_{2}$ such that $\llbracket$ every $\mathbf{P}$ is a $\mathbf{Q} \rrbracket^{\left\langle D, I_{1}\right\rangle} \neq$ $\llbracket$ every $\mathbf{P}$ is a $\mathbf{Q} \rrbracket^{\left\langle D, I_{2}\right\rangle}$. Hence, (23) does not come out as L-analytical. This is expected, as this sentence is a non-systematic tautology.

On the other hand, consider the interpretation of the LS in (24), given in (26) below. Following Barwise and Cooper (1981), it is assumed that there simply denotes the domain of individuals $D_{e}$.

$$
\begin{aligned}
\llbracket \text { there is every } \mathbf{P} \rrbracket^{\langle D, I\rangle} & =\llbracket \text { every } \rrbracket(I(\mathbf{P}))\left(\llbracket \text { there } \rrbracket^{\langle D, I\rangle}\right) \\
& =\llbracket \text { every } \rrbracket^{\langle D, I\rangle}(I(\mathbf{P}))\left(D_{e}\right)
\end{aligned}
$$

It isn't possible to find an interpretation $I$ such that $\llbracket$ there is every $\mathbf{P} \rrbracket^{\langle D, I\rangle}=0$, because $I(\mathbf{P}) \subseteq D_{e}$ for all $I$. This means that, as expected, (24) comes out as L-analytical, which accounts for its ungrammaticality.

\subsubsection{Capturing the anti-rogativity of neg-raising predicates in terms of L-analyticity}

Let us now return to the selectional restrictions of neg-raising predicates and see how the account sketched in Sect. 3.1.3 can be made fully explicit by phrasing it in terms of L-analyticity. In order to do so, two assumptions about the structure of interrogative clauses and neg-raising predicates are needed.

Interrogative clauses are headed by a question operator. Firstly, we asssume that interrogative clauses are headed by a question operator, written as '?'. Semantically, this operator takes the semantic value of its prejacent $P$ as its input, and yields $P \cup \neg P$ as its output:

$$
\llbracket ? \rrbracket^{w}=\lambda P_{T} \cdot P \cup \neg P
$$

In terms of alternatives, ? adds to the alternatives already contained in $P$ one additional alternative, which is the set-theoretic complement of the union of all the alternatives in $P$. This is a standard operation in inquisitive semantics (see, e.g., Ciardelli et al. 2015). Note that it always results in a set of alternatives which together cover the entire logical space, i.e., a sentence meaning that is non-informative. ${ }^{20}$

Lexical decomposition of neg-raising predicates. Secondly, we assume that a negraising predicate $V$ is decomposed at LF into two components, $R_{E M}$ and $\mathcal{M}_{V}$, the former of which but not the latter is a logical item in the relevant sense. While $R_{E M}$ is common to all neg-raising predicates, $\mathcal{M}_{V}$ is specific to the predicate $V .{ }^{21} \mathrm{An} \mathrm{LF}$ in which believe is decomposed into these two components is given in (28).

\footnotetext{
20 The exact treatment of the question operator does not really matter for our purposes. The only thing that is crucial is that it always results in non-informativity. In particular, our account is also compatible with a treatment of the question operator under which it (i) only adds an additional alternative if its input $P$ is not yet inquisitive, and (ii) adds a presupposition to the effect that at least one of the alternatives in its output is true (Roelofsen 2013b).

21 Bošković and Gajewski (2011) propose a very similar decomposition of neg-raising predicates, motivated on independent grounds.
} 
(28)

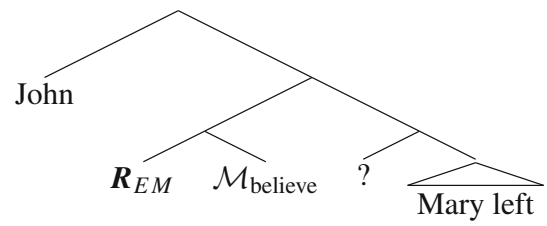

The non-logical component, $\mathcal{M}_{V}$, is a function that maps an individual $x$ to a modal base. Which modal base this is gets determined by the predicate $V$. In the case of, e.g., believe, it is $x$ 's doxastic state, while in the case of want it is $x$ 's bouletic state:

$$
\begin{aligned}
& \text { a. } \llbracket \mathcal{M}_{\text {believe }}(x) \rrbracket^{w}=\operatorname{DOX}_{x}^{w} \\
& \text { b. } \llbracket \mathcal{M}_{\text {want }}(x) \rrbracket^{w}=\operatorname{BOUL}_{x}^{w}
\end{aligned}
$$

The logical component, $R_{E M}$, does two things: it triggers the EM presupposition and acts as compositional glue by connecting $\mathcal{M}_{V}$ to the subject and the complement:

$$
\llbracket R_{\mathrm{EM}} \rrbracket=\lambda \mathcal{M}_{\langle\mathrm{e}, \mathrm{st}\rangle} \cdot \lambda P_{\langle s t, t\rangle} \cdot \lambda x: \underline{\mathcal{M}}(x) \in P \vee \mathcal{M}(x) \in \rightarrow P . \mathcal{M}(x) \in P
$$

$R_{E M}$ takes the function $\mathcal{M}_{V}$, the complement meaning $P$, and the subject $x$ as arguments; it contributes the EM presupposition (the modal base $\mathcal{M}_{V}(x)$ has to be a resolution either of $P$ or of the negation of $P$ ); and it asserts that $\mathcal{M}_{V}(x)$ is a resolution of $P$. Intuitively, $R_{E M}$ is a logical item because it does not contribute any "contingent content" of its own: its denotation, in contrast to that of $\mathcal{M}_{V}$, does not vary between models.

L-analyticity. We now have all the ingredients needed to show that the trivial sentence meanings we identified in Sect. 3.1.3 are L-analytical. There, we had found that whenever a neg-raising attitude predicate like believe combines with an interrogative complement, as in (31), its asserted content is trivial relative to its presupposition.

*John believes whether Mary left.

Let's start by constructing the LS for (31): the subject, the complement clause, and the function $\mathcal{M}_{\text {believe }}$ each get substituted by a fresh constant, while both $R_{E M}$ and the interrogative marker remain untouched.
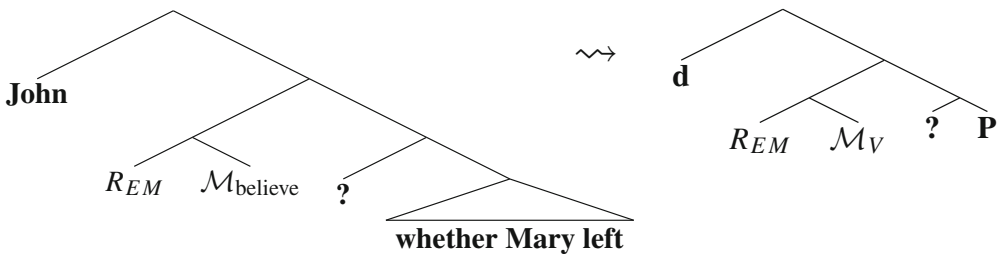

The denotation of this LS is given in (33a), its presupposition in (33b).

$$
\begin{array}{llll}
\text { a. } & \text { Asserted content: } & \llbracket \mathcal{M}_{V}(\mathbf{d}) \rrbracket \in \llbracket \mathbf{P P} \rrbracket & \\
\text { b. } & \text { Presupposition: } & \llbracket \mathcal{M}_{V}(\mathbf{d}) \rrbracket \in \llbracket \mathbf{P P} \rrbracket \text { or } & \llbracket \mathcal{M}_{V}(\mathbf{d}) \rrbracket \in \llbracket \rightarrow \mathbf{P P} \rrbracket
\end{array}
$$

First, we note that the first disjunct in the presupposition is identical with the asserted content. Next, let's look at the second disjunct in the presupposition. We find that, 
no matter what $\mathbf{P}$ is, the set of propositions in $\llbracket \mathbf{P} \mathbf{P} \rrbracket^{\langle D, I\rangle}$ covers the entire logical space. Hence, we also know that $\llbracket \neg \mathbf{? P} \rrbracket^{\langle D, I\rangle}=\{\emptyset\}$ for all $I$. The second disjunct in the presupposition is thus only satisfied if $\llbracket \mathcal{M}_{V}(\mathbf{d}) \rrbracket^{\langle D, I\rangle}=\emptyset$. But observe that, whenever this holds, then the first disjunct is also satisfied. This is because every sentence meaning contains the inconsistent proposition, which means that $\llbracket \mathbf{P} \mathbf{P} \rrbracket^{\langle D, I\rangle}$ contains $\emptyset$, regardless of what $\mathbf{P}$ is. Thus, whenever the second disjunct holds, the first one holds as well, or, in other words, whenever the presupposition is satisfied, the first disjunct is true.

Now, since the first disjunct, as noted initially, is identical with the asserted content, this in turn means that, for all interpretations in which the denotation of the LS is defined, this denotation will be 1 . Sentence (31) hence comes out as L-analytical, which is what we set out to show.

Anti-rogativity and the defeasibility of neg-raising. Finally, let us return to a case in which the neg-raising inference is suspended, repeated in (34) below.

Bill doesn't know who killed Caesar. He isn't even sure whether or not Brutus and Caesar lived at the same time. So, naturally...

Bill doesn't believe that Brutus killed Caesar.

y $\rightarrow$ Bill believes that Brutus didn't kill Caesar.

One might expect that in such contexts, since the neg-raising inference of the predicate does not really surface, the incompatibility with interrogative complements will also be lifted. This is not the case, however. As witnessed by (35), interrogative complements are still unacceptable in such configurations.

Bill doesn't know who killed Caesar. He isn't even sure whether or not Brutus and Caesar lived at the same time. So, naturally...

*Bill doesn't believe whether Brutus killed Caesar.

This is correctly predicted. Recall that according to Gajewski's (2009) theory of Lanalyticity, for a sentence to be perceived as ungrammatical it is sufficient that a constituent of its logical form is L-analytical. This is indeed the case in (35): even though the full sentence is not L-analytical (assuming that the EM presupposition is locally accommodated), the clause that gets negated (Bill believes whether Brutus killed Caesar) is L-analytical. This is sufficient to account for the perceived ungrammaticality.

\subsection{Truth-evaluating predicates: be true and be false}

We have seen above how the selectional restrictions of a substantial class of antirogative predicates, namely those that are neg-raising, can be derived. We now turn to another, much smaller class of anti-rogatives consisting of the truth-evaluating predicates be true and be false.

Recall that the basic entry for believe requires that $\operatorname{DOX}_{x}^{w} \in P$, where $x$ is the subject of the predicate, $w$ the world of evaluation, and $P$ the semantic value of the 
complement. The requirement says that $\operatorname{DOX}_{x}^{w}$, the information state of $x$ in $w$, should be a resolution of $P$. Many other attitude predicates can be treated similarly, replacing $\operatorname{DOX}_{x}^{w}$ by another appropriate modal base associated with the individual $x$.

At first sight, be true and be false do not fit this mold, since they do not involve an individual subject, let alone make reference to any modal base associated with such an individual. Yet it is possible to view be true and be false in a way that is quite similar to the above view on believe and other attitude predicates. Namely, even though it does not make sense to explicate the semantics of be true and be false in terms of a modal base associated with a particular individual, it is natural to think of these predicates in terms of a modal base that depends only on the world of evaluation $w$. Let us denote this modal base as TRUE ${ }^{w}$. Given a world $w$, what should TRUE ${ }^{w}$ be? In view of the truth-evaluating function of be true and be false, it is natural to require that $\mathrm{TRUE}^{w}$ should determine exactly what is true and what is false in $w$. But this simply means that TRUE ${ }^{w}$ should be the singleton set $\{w\}$. Viewed as a doxastic state, this is a state of complete information, according to which the only candidate for the actual world is $w$.

Using TRUE ${ }^{w}$ as the relevant modal base, we can now give lexical entries for be true and be false which are structurally parallel to our basic entry for believe. As expected, the only difference is that be true and be false do not take an individual subject as one of their arguments, and accordingly the modal base that they rely on does not depend on such an individual.

$$
\begin{aligned}
& \text { a. } \llbracket \text { be true } \rrbracket^{w}=\lambda P_{T} \cdot \operatorname{TRUE}^{w} \in P \\
& \text { b. } \llbracket \text { be false } \rrbracket^{w}=\lambda P_{T} \cdot \operatorname{TRUE}^{w} \notin P
\end{aligned}
$$

When combined with a declarative complement, these entries give the expected results. For instance, $\llbracket$ It is true that Ann left $\rrbracket^{w}=1$ just in case $\{w\} \in \llbracket$ Ann left $\rrbracket$, which means that $w$ must be a world in which Ann left. Similarly, $\llbracket$ It is false that Ann left $\rrbracket^{w}=1$ just in case $\{w\} \notin \llbracket$ Ann left $\rrbracket$, which means that $w$ must be a world in which Ann didn't leave.

Now, what happens when be true and be false take an interrogative complement? We have seen in Sect. 2 that if $P$ is the semantic value of an interrogative complement, its elements cover the entire logical space, i.e., $\bigcup P=W$. Since sentence meanings are downward closed, this means that $\{w\} \in P$ for any $w \in W$. This makes sense: a question is always resolved by a doxastic state that contains full information as to what the world is like. But this means that, if $P$ is the semantic value of an interrogative complement, 【be true $\rrbracket^{w}(P)=1$ and $\llbracket$ be false $\rrbracket^{w}(P)=0$ for any $w \in W$. Hence, when taking interrogative complements, be true and be false systematically yield a tautology and a contradiction, respectively. Assuming that be true and be false constitute logical vocabulary, these are again cases of L-analyticity. This provides an explanation for why truth-evaluating predicates don't accept interrogative complements. 


\section{Rogative predicates}

We now turn to rogative predicates. This class includes predicates such as wonder and be curious, which Karttunen (1977) calls 'inquisitive predicates', as well as predicates of dependency such as depend on and be determined by, and speech act predicates such as ask and inquire. We focus here on the first two subclasses and will briefly remark on the third in the conclusion.

\subsection{Inquisitive predicates}

Ciardelli and Roelofsen (2015) and Uegaki (2015b) offer an account of the selectional restrictions of wonder. The former is couched within the same general approach to clause embedding that we are assuming here, i.e., one in which declarative and interrogative complements are assumed to be of the same semantic type. We briefly review this account here, adapting it to our current terminology. The account can, with small modifications, be extended to other inquisitive predicates such as be curious and investigate. ${ }^{22}$ For discussion of the subtle differences between the accounts of Ciardelli and Roelofsen (2015) and Uegaki (2015b), respectively, we refer to Appendix A.2.

To model what it means for an individual to wonder, we first need a representation of the issues that she entertains. Ciardelli and Roelofsen call this her inquisitive state. Formally, an individual's inquisitive state in $w, \mathrm{INQ}_{x}^{w}$, is a downward-closed set of consistent propositions which together cover her doxastic state, i.e., $\bigcup \mathrm{INQ}_{x}^{w}=\mathrm{DOX}_{x}^{w}$. The propositions in $\mathrm{INQ}_{x}^{w}$ are those that are informative enough to resolve the issues that $x$ entertains. They correspond to extensions of her current doxastic state in which all her questions are settled one way or another.

Informally, $x$ wonders about a question, e.g., about who called, just in case (i) $x$ isn't certain yet who called, and (ii) she wants to find out who did. This is the case exactly if (i) $x$ 's current doxastic state does not resolve the question, and (ii) every doxastic state in $x$ 's inquisitive state is one that does resolve the question:

$$
\llbracket \text { wonder } \rrbracket^{w}=\lambda P_{T} \cdot \lambda x . \underbrace{\mathrm{DOX}_{x}^{w} \notin P}_{x \text { isn't certain yet... }} \wedge \underbrace{\mathrm{INQ}_{x}^{w} \subseteq P}_{\text {but wants to find out }}
$$

This entry yields desirable results when the predicate takes an interrogative complement. Now let us consider what happens when it takes a declarative complement:

*John wonders that Mary called.

Recall that if $P$ is the meaning of a declarative complement it always contains a single alternative $\alpha$. Since complement meanings are downward-closed, this means that $P$ amounts to the powerset of $\alpha, \wp(\alpha)$. Now suppose that the first conjunct in (37) holds: $\operatorname{DOX}_{x}^{w} \notin P$. Then it must be that $\operatorname{DOX}_{x}^{w} \nsubseteq \alpha$. But then, since $\bigcup \mathrm{INQ}_{x}^{w}=\mathrm{DOX}_{x}^{w}$, it must also be that $\bigcup \mathrm{INQ}_{x}^{w} \nsubseteq \alpha$. It follows that there is at least one $s \in \mathrm{INQ}_{x}^{w}$ such that $s \nsubseteq \alpha$. But if $s \nsubseteq \alpha$, then since $\alpha$ is the unique alternative in $P$, we have that $s \notin P$. So the

\footnotetext{
22 Crucially, be curious is like wonder and unlike the closely related (but responsive) predicate be of interest in that it implies ignorance.
} 


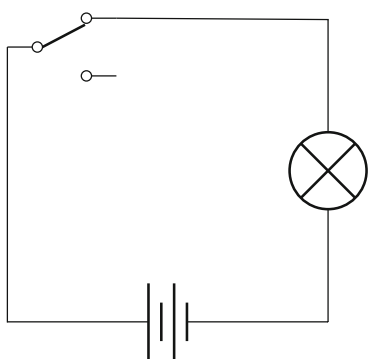

(a) Full dependency

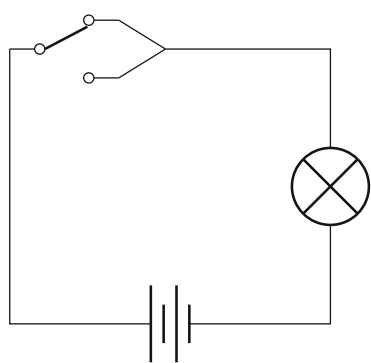

(b) Trivial dependency

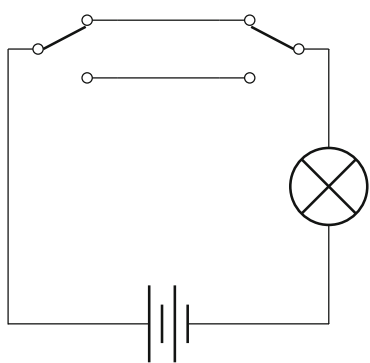

(c) Partial dependency

Fig. 3 Full, trivial, and partial dependency between (left) switch and light

second conjunct in the lexical entry must be false. Hence, whenever wonder takes a declarative complement, this results in a contradictory sentence meaning.

\subsection{Verbs of dependency}

We now turn to rogative predicates of dependency, such as depend on and be determined by (on one of its interpretations). We will concentrate on depend on, but it seems that the account we will present could be straightforwardly extended to other predicates of dependency.

Our treatment of depend on builds on that of Ciardelli (2016, p.243), who argues that dependency statements are modal statements. One can only sensibly say that one thing depends on another relative to some specific range of relevant possible worlds, i.e., a modal base. This modal base can either be explicitly given, as in (39), or inferred from the context, as in (40), where, roughly, it is construed as 'given the laws of nature and the electrical circuit under discussion'.

According to Dutch law, one's income tax rate depends on one's age.

To form an intuition about what it means for one thing to depend on another, let us focus on example (40) and consider the electrical circuit in Fig. 3a. Let $w_{1}$ be the actual world, in which the switch is up and the light on, and let $w_{2}$ be a world in which the switch is down and the light off. The modal base $\sigma_{w_{1}}$ consists of all worlds in which the laws of nature are the same as in $w_{1}$ and in which the circuit is exactly as given in Fig. 3a. That is, $\sigma_{w_{1}}=\left\{w_{1}, w_{2}\right\}$. Let $P_{\text {light }}$ be the meaning of the first argument of the predicate in (40), whether the light is on, and $P_{\text {switch }}$ the meaning of the second argument, whether the switch is up. What does it mean for $P_{\text {light }}$ to depend on $P_{\text {switch }}$ relative to $\sigma_{w_{1}}$ ?

On a first approximation, it means that whenever we rule out enough possible worlds in our modal base to establish some alternative in $P_{\text {switch }}$, we also automatically establish some alternative in $P_{\text {light }}$. That is, whenever we determine whether the switch is up or down, it is also determined whether the light is on or off. More generally, we could say that $P$ depends on $P^{\prime}$ relative to a modal base $\sigma$ if and only if there is a 
function $f$ that maps each alternative $\alpha \in \operatorname{alt}\left(P^{\prime}\right)$ to an alternative $f(\alpha) \in \operatorname{alt}(P)$ such that for all $p \subseteq \sigma$, if $p \subseteq \alpha$ for some $\alpha \in$ alt $\left(P^{\prime}\right)$ then $p \subseteq f(\alpha)$ as well. This is the logical notion of dependency that Ciardelli (2016) proposes and investigates.

We will further refine this basic notion, however, in order to rule out trivial dependencies, i.e., cases in which the function $f$ maps every alternative in $\operatorname{alt}\left(P^{\prime}\right)$ that is compatible with $\sigma$ to the same alternative in alt $(P)$. To see that such cases need to be ruled out, suppose that the light is always on, no matter whether the switch is up or down, as in the circuit in Fig. 3b. Let $w_{3}$ be the actual world in this scenario-i.e, the world in which the switch is up and the light on-and let $w_{4}$ be a world in which the switch is down but the light still on. Then we have that $\sigma_{w_{3}}=\left\{w_{3}, w_{4}\right\}$. In this scenario, it is certainly still possible to find a function $f$ mapping every alternative $\alpha$ in alt $\left(P_{\text {switch }}\right)$ to some alternative $f(\alpha)$ in alt $\left(P_{\text {light }}\right)$ such that for all $p \subseteq \sigma_{w_{3}}$, if $p \subseteq \alpha$ for some $\alpha \in \operatorname{alt}\left(P^{\prime}\right)$ then $p \subseteq f(\alpha)$ as well. Just let $f$ map both alternatives in $\operatorname{alt}\left(P_{\text {switch }}\right)$ to the same alternative in alt $\left(P_{\text {light }}\right)$, namely the alternative "that the light is on'. But we would not say that sentence (40) is true in this scenario. Whether the light is on does not depend on whether the switch is up. It's just always on. So, we should require that the function $f$ does not map all the alternatives in $\operatorname{alt}\left(P_{\text {switch }}\right)$ that are compatible with $\sigma_{w_{3}}$ to the same alternative in alt $\left(P_{\text {light }}\right)$. This leads us to the following entry for depend on: ${ }^{23}$

$$
\begin{aligned}
& \text { 匹depend on } \rrbracket^{w}=\lambda P_{T}^{\prime} \cdot \lambda P_{T} \cdot \exists f \in \operatorname{alt}(P)^{\text {alt }\left(P^{\prime}\right)} \text { such that: } \\
& \text { (i) } \forall p \subseteq \sigma_{w} \cdot \forall \alpha \in \operatorname{alt}\left(P^{\prime}\right) .(p \subseteq \alpha \rightarrow p \subseteq f(\alpha)) \text { and } \\
& \text { (ii) } \left.\exists \alpha, \alpha^{\prime} \in \operatorname{alt}\left(P^{\prime}\right) \cdot \alpha \cap \sigma_{w} \neq \emptyset \wedge \alpha^{\prime} \cap \sigma_{w} \neq \emptyset \wedge f(\alpha) \neq f\left(\alpha^{\prime}\right)\right)
\end{aligned}
$$

Now let us examine whether this lexical entry accounts for the selectional restrictions of the predicate. What happens if either the first or the second argument of the predicate is a declarative clause? First consider the following case:

*That the light is on depends on whether the switch is up.

In this case, $P$ contains a single alternative. This means that it will be impossible to find a function $f \in \operatorname{alt}(P)^{\text {alt }\left(P^{\prime}\right)}$ that satisfies condition (ii) in the entry above, i.e., one that does not map every element of alt $\left(P^{\prime}\right)$ onto the same element of alt $(P)$. Thus, (42) comes out as a contradiction, and this will always be the case if the first argument of the predicate is a declarative clause.

Now consider a case in which the second argument is a declarative clause: ${ }^{24}$

\footnotetext{
23 This entry may be further refined in order to allow for partial dependencies. For instance, in the circuit in Fig. 3c, whether the light is on only partially depends on the position of the switch on the left. The position of the switch on the right now also matters. On a first approximation, we could say that $P$ partially depends on $P^{\prime}$ if we can find a third sentence meaning $P^{\prime \prime}$ such that $P$ fully depends on $P^{\prime} \cap P^{\prime \prime}$ but not on $P^{\prime \prime}$ alone (cf., Karttunen 1977, fn.6). We do not explicitly work out this refinement here, because it would not yield different predictions about the selectional restrictions of depend on.

24 As noted by an anonymous reviewer, the reader might wonder whether the ungrammaticality of (43) isn't rooted in syntax rather than semantics. After all, declarative complements generally do not combine with prepositions in English (unlike interrogative complements). However, as the same reviewer notes, a semantic explanation does seem to be needed, for at least two reasons. First, movement (e.g., topicalization)
} 
(43) *Whether the light is on depends on that the switch is up.

In this case, $P^{\prime}$ contains a single alternative. This again means that it will be impossible to find a function $f \in \operatorname{alt}(P)^{\text {alt }\left(P^{\prime}\right)}$ that satisfies condition (ii) in the entry of the predicate. So (43) also comes out as a contradiction, and the same result obtains if the predicate takes other declarative clauses as its second argument. This systematic contradictoriness explains why depend on cannot take declarative complements. ${ }^{25}$

\section{Empirical and methodological challenges}

In this final section we will identify an empirical challenge for the account laid out above. We will show that, at least in the case of neg-raising predicates, this challenge can be addressed. However, doing so will bring out a general methodological issue for semantic accounts of ungrammaticality.

\subsection{Empirical challenge: mixed complements}

The account presented above makes incorrect predictions for the case of mixed complements, i.e., complex complements formed by conjoining a declarative and an interrogative clause. As illustrated in (44), anti-rogative and rogative predicates do not accept mixed complements. Our account, however, predicts the examples in (44) to be grammatical.

Footnote 24 continued

generally resolves the incompatibility between declarative complements and prepositions, as in (i), but this is not the case for depend on, as seen in (ii):

(i) a. *They complained about that Mary left.

b. That Mary left is what they complained about.

(ii) a. *Your salary depends on that you have a PhD.

b. *That you have a PhD is what your salary depends on.

Second, looking beyond English, there are languages in which declarative complements can in principle combine with prepositions, but are still ungrammatical under depend on. A case in point is Spanish, as illustrated in (iii) and (iv).

(iii) Estoy convencido de que podemos trabajar juntos.

I-am convinced of that we-can work together

'I am convinced that we can work together.'

(iv) $* \mathrm{Si}$ podemos trabajar juntos depende de que tenemos la misma ética. Whether we-can work together depends on that we-have the same ethics 'Whether we can work together depends on that we have the same ethics.'

25 Notice that there is an interesting similarity between our entry for depend on and that for wonder: the first condition in the entry for depend on is similar to the 'entertain' condition in the entry for wonder, and the second condition in the entry for depend on is similar to the 'ignorance' condition in the entry for wonder. 

a. *John believes/thinks that Mary left and when she did.
b. *It is true that Mary left and when she did.
c. *John wonders that Mary left and when she did.

To see why we make these predictions, recall that a declarative clause is usually informative, i.e., $\operatorname{info}(\varphi) \subset W$, and that an interrogative clause is usually inquisitive, i.e., $\operatorname{info}(\varphi) \notin \llbracket \varphi \rrbracket$. To compute the meaning of two conjoined clauses, we simply take their intersection, i.e., $\llbracket \varphi \wedge \psi \rrbracket=\llbracket \varphi \rrbracket \cap \llbracket \psi \rrbracket$. This means that, if one of the conjoined clauses is informative, then so is the conjunction as a whole, and if one of the conjuncts is inquisitive, then the conjunction as a whole is typically inquisitive as well. For example, the mixed complement in (44), that Mary left and when she did, is both informative and inquisitive.

Now, let's focus on the case of neg-raising predicates. On our account, it is the noninformativity of interrogative complements that leads to systematic triviality whenever these complements appear under neg-raising predicates. Declarative complements don't give rise to such systematic triviality because they are typically informative. More concretely, recall that if believe (its lexical entry is repeated below in (45)) takes an interrogative complement $P$, then the second disjunct of the EM presupposition makes a vacuous contribution to the disjunction as a whole, since $\rightarrow P=\{\varnothing\}$. By contrast, if believe takes a declarative complement with meaning $P$, the second disjunct makes a non-vacuous contribution, since $\rightarrow P \neq\{\emptyset\}$. A mixed complement, because it is informative, behaves just like a declarative complement in this respect, hence averting triviality. This means that believe is wrongly predicted to accept mixed complements.

$$
\llbracket \text { believe } \rrbracket^{w}=\lambda P_{T} \cdot \lambda x: \operatorname{DOx}_{x}^{w} \in P \vee \operatorname{DOx}_{x}^{w} \in \rightarrow P . \operatorname{DOx}_{x}^{w} \in P
$$

Below we suggest an alternative way of formulating the EM presupposition, which makes correct predictions for mixed complements.

\subsection{Projection operators}

To formulate a suitable version of the EM presupposition, we first introduce a number of operators on sentence meanings, familiar from inquisitive semantics as projection operators. The !-operator eliminates inquisitiveness: $! P$ is always non-inquisitive. The ?-operator, already familiar from our treatment of interrogative complements in Sect. 3, eliminates informativity: ?P is always non-informative. Finally, we introduce the $\langle$ ? $\rangle$-operator from Roelofsen (2015), which can be thought of as a conditional variant of the ?-operator: if $P$ is inquisitive, then $\langle ?\rangle$ has no effect, but if $P$ is not inquisitive, then $\langle ?\rangle P=? P$.

$$
\begin{aligned}
& ! P=\cup P \\
& ? P=P \cup \rightarrow P \\
& \langle ?\rangle P=\left\{\begin{array}{ll}
? P \text { if } P \text { is not inquisitive } \\
P \text { otherwise }
\end{array}\right\}
\end{aligned}
$$


The $\langle$ ? $\rangle$-operator ensures inquisitiveness while preserving other semantic properties of its prejacent, in particular its informative content and its decision set, ${ }^{26}$ as much as possible. In Roelofsen (2015) and Ciardelli et al. (2018) this operator is taken to play an important role in the interpretation of interrogative clause type marking.

\subsection{Reformulating the EM presupposition}

We will now first formulate the EM presupposition in terms of the ?-operator. This will be just a notational variant of our old formulation. In a second step, we will then formulate the EM presupposition in terms of the $\langle ?\rangle$-operator. This re-formulation will yield the same results for declarative and interrogative complements, but will make a difference for mixed complements.

Note that, for any proposition $p$ and sentence meaning $P$, the condition that $p \in$ $P \vee p \in \rightarrow P$ is equivalent to $p \in ? P$. Using this equivalence, we can reformulate our lexical entry for believe as follows:

$$
\llbracket \text { believe } \rrbracket^{w}=\lambda P_{T} \cdot \lambda x: \underline{\operatorname{DOx}_{x}^{w} \in ? P} . \operatorname{DOx}_{x}^{w} \in P
$$

Now, let's see what happens if we define the EM presupposition in terms of the $\langle ?\rangle$ operator instead of the ?-operator:

$$
\llbracket \text { believe } \rrbracket^{w}=\lambda P_{T} \cdot \lambda x: \operatorname{DOx}_{x}^{w} \in\langle ?\rangle P . \operatorname{DOx}_{x}^{w} \in P
$$

If believe takes a declarative complement, then $P$ is not inquisitive. This means that the $\langle$ ? $\rangle$-operator contributes the same meaning as the ?-operator, and the lexical entry for believe amounts to (49). As we have just seen, this formulation is equivalent to our original lexical entry for believe. So, in the case of declarative complements, nothing has changed.

(49) $\llbracket$ believe $\rrbracket^{w}=\lambda P_{T} \cdot \lambda x: \operatorname{DOx}_{x}^{w} \in ? P . \operatorname{DOx}_{x}^{w} \in P \quad$ [with non-inquisitive complement]

If believe takes an interrogative complement, then $P$ is inquisitive. This means that the $\langle$ ? $\rangle$-operator doesn't have any effect and the lexical entry for believe reduces to (50). In other words, the presupposition and the asserted content are identical. So, in the case of interrogative complements we derive the same triviality as before.

$$
\llbracket \text { believe } \rrbracket^{w}=\lambda P_{T} . \lambda x: \underline{\text { DOx }_{x}^{w} \in P} . \text { DOx }_{x}^{w} \in P \quad \text { [with inquisitive complement] }
$$

Finally, if believe takes a mixed complement, then $P$ is also inquisitive, and with the same reasoning as for interrogative complements, this configuration results in triviality. With the modified version of the EM presupposition, we hence correctly predict that believe doesn't accept mixed complements.

\footnotetext{
26 The decision set of a sentence $\varphi$ with meaning $P$ is the set of propositions that either resolve the issue expressed by $\varphi$ or establish that this issue cannot be truthfully resolved, i.e., the set of propositions $P \cup \neg P$.
} 
Note that while this modification of our account solves the mixed complement problem for neg-raising predicates, the problem persists for other anti-rogative and rogative predicates. ${ }^{27}$

\subsection{A methodological note}

As we have just seen, one way of formulating the EM presupposition made the right predictions for mixed complements, while another formulation didn't. This brings out a general limitation of semantic accounts of ungrammaticality. The problem, as we see it, is that we cannot distinguish between the two formulations of the EM presupposition on independent grounds. This is because they make exactly the same predictions for declarative complements - and the case of declarative complements is the only one where we can check whether our account derives the correct meaning. In all the other cases, we cannot check this because the sentences are ungrammatical and therefore simply have no "observable" semantic properties. So, while for neg-raising predicates we have independent motivation for assuming an EM presupposition per se (namely, we can observe that these predicates are neg-raising), there is no independent motivation for preferring any particular formulation of the EM presupposition. Thus, it cannot be said that the account given here fully derives the selectional restrictions of predicates like believe and think from independently observable semantic properties of these predicates, i.e., the fact that they are neg-raising. Rather, we have shown that making one particular assumption about the lexical semantics of these predicates, namely that they involve an EM presupposition formulated in terms of $\langle$ ? $\rangle$, accounts both for their neg-raising property and for their selectional restrictions.

As far as we can see, this is a principled limitation affecting all semantic accounts of ungrammaticality. All such accounts have to rely on specific lexical entries for the expressions involved. It is often possible to motivate these lexical entries on independent grounds, in the sense that they make good predictions for grammatical cases. However, it is often difficult, if not impossible, to show that these entries could not be altered in such a way that the good predictions about the grammatical cases would be preserved while the ungrammatical cases would no longer come out as trivial.

\section{Conclusion}

There are two kinds of approaches to the semantics of clausal complements, one that assumes different types for declarative and interrogative complements and one that assumes uniform typing. On the first approach, the selectional restrictions of clauseembedding predicates can to some extent be accounted for in terms of a type mismatch, but in the absence of independent motivation for the assumed type distinction and the type requirements of the relevant predicates, such an account remains stipulative.

\footnotetext{
27 In the case of truth-evaluating predicates, there is a natural way to address the problem. Namely, if such predicates take a mixed complement, the interrogative conjunct will always be redundant, in the sense that leaving it out would not affect the interpretation of the sentence as a whole. Such redundancy is known to manifest itself as unacceptability (see, e.g., Schlenker 2009; Katzir and Singh 2013; Mayr and Romoli 2016).
} 
On the second approach, the selectional restrictions of clause-embedding predicates have to be explained entirely based on semantic properties of the relevant predicates. Extending initial work of Ciardelli and Roelofsen (2015) and Uegaki (2015b), we have seen in this paper that such an explanation can be given for several important classes of rogative and anti-rogative predicates, namely neg-raising predicates, truth-evaluating predicates, inquisitive predicates, and predicates of dependency.

Cases that we have not treated here include rogative speech act predicates such as ask and inquire, anti-rogative speech act predicates such as assert and claim, as well as non-veridical preferential predicates like fear and desire. The selectional restrictions of this last class of predicates have been addressed elegantly in recent work by Uegaki and Sudo (2017).

For rogative speech act predicates such as ask and inquire, we might attempt a simple explanation along the following lines. It is natural to assume that part of what a sentence like $x$ asked $\varphi$ conveys is that $x$ uttered a sentence $\varphi$ which was inquisitive w.r.t. the common ground in the context of utterance. Arguably, this is necessary in order to satisfy the sincerity conditions of the speech act of asking, and similarly for inquiring. This requirement cannot be met if $\varphi$ is a declarative, because in that case it is bound to be non-inquisitive w.r.t. the common ground.

For anti-rogative speech act predicates like assert and claim, we believe that an explanation is harder to find. This is because there are closely related speech act predicates such as announce, state, and tell which are responsive. If we tried to appeal to a similar reasoning as with rogative speech act predicates, we would have to motivate why this reasoning applies to predicates like assert, but not to predicates like announce. Instead, following White and Rawlins (2016), we conjecture that the relevant factor determining whether an 'assertive' speech act predicate is responsive or anti-rogative might lie in the predicate's event structure. Further exploring this hypothesis, however, must be left for another occasion.

Moreover, while all predicates we discussed here could easily be classified as either responsive or (anti-)rogative, not all embedding predicates fall so neatly into one of these categories. One complication stems from the fact that the selectional restrictions of some predicates appear to be polarity sensitive (Mayr 2017). For instance, as illustrated in (51) and (52), say and be certain seem to allow whether-complements when appearing under negation, but not when appearing in positive episodic sentences.

a. Mary didn't say whether Bill had eaten.

b. *Mary said whether Bill had eaten.

a. Mary isn't certain whether Bill has eaten.

b. *Mary is certain whether Bill has eaten.

Mayr (2017) proposes that the environments in which verbs like say and be certain accept whether-complements are exactly the same environments in which NPIs are licensed. In recent experimental work, van Gessel et al. (2018) found confirmation for the polarity sensitivity of be certain whether, but could not confirm Mayr's hypothesis that this construction is acceptable exactly in those environments that license NPIs: acceptability judgments for be certain whether do not correlate with judgments on NPIs. 
Another complication, illustrated in (53), is that certain predicates, namely emotive factives like surprise and amaze, only accept $w h$-interrogatives as complements, but not polar interrogatives.

a. It is amazing what Bill had for breakfast.

b. *It is amazing whether Bill had breakfast.

Several accounts of this phenomenon have been suggested (d'Avis 2002; Abels 2004; Guerzoni 2007; Sæbø 2007; Nicolae 2013; Romero 2015). For a detailed overview of this literature, as well as a proposal that is directly compatible with the account developed in the present paper, we refer to Roelofsen 2017 and Roelofsen et al. 2019.

Open Access This article is distributed under the terms of the Creative Commons Attribution 4.0 International License (http://creativecommons.org/licenses/by/4.0/), which permits unrestricted use, distribution, and reproduction in any medium, provided you give appropriate credit to the original author(s) and the source, provide a link to the Creative Commons license, and indicate if changes were made.

\section{Appendix A. Related work}

This appendix discusses some work that is, like the present paper, concerned with the selectional restrictions of rogative and/or anti-rogative predicates. In particular, we will consider the work of Zuber (1982), Egré (2008), Mayr (2017), and Cohen (2017a,b) on the connection between anti-rogativity and neg-raising (Sect. A.1), and the work of Uegaki (2015b) on the selectional restrictions of wonder and possible independent motivation for a type distinction between anti-rogatives on the one hand and rogatives and responsives on the other (Sect. A.2).

\section{A.1 On the connection between anti-rogativity and neg-raising}

Evidently, the discussion of anti-rogativity in the present paper is greatly indebted to Zuber (1982), who observed the connection between anti-rogativity and neg-raising. Zuber's work was brought to our attention through the insightful discussion of clausal embedding in Egré (2008). However, neither Zuber (1982) nor Egré (2008) succeeded in deriving anti-rogativity from neg-raising in a principled way.

Independently of the present paper, Mayr (2017) and Cohen (2017a,b) have also recently proposed ways to explain the connection between anti-rogativity and negraising. ${ }^{28}$ While these accounts are largely in the same spirit as ours, they are more limited in scope and less explicit in some important regards. We will discuss each account in some more detail below.

\section{A.1.1 Mayr (2017)}

Mayr (2017) assumes the following lexical entry for believe:

28 As mentioned, a first version of the present account started circulating in the spring of 2016. 


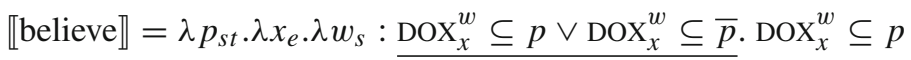

That is, just as we did, he incorporates an excluded-middle presupposition to capture the fact that believe is neg-raising, following Gajewski (2007). However, he assumes that anti-rogative predicates like believe and responsive predicates like know and be certain all take a single proposition as their first input, while we take them to apply to sets of propositions.

Mayr takes a declarative complement to denote a single proposition, so such a complement can straightforwardly combine with believe, as well as with know and be certain. A polar interrogative complement, on the other hand, is taken to denote a kind of type-raised existential quantifier over sets of propositions. For instance, whether Mary smokes is interpreted as follows: ${ }^{29}$

$$
\begin{aligned}
& \llbracket \text { whether Mary smokes } \rrbracket=\lambda Q_{\langle s t, s t\rangle} \cdot \lambda w_{s} \cdot \exists p \in Q^{\prime} \cdot Q(p)(w)=1 \\
& \text { where } Q^{\prime}=\left\{\lambda w^{\prime} \text {. Mary smokes in } w^{\prime}, \lambda w^{\prime} \text {.Mary doesn't smoke in } w^{\prime}\right\}
\end{aligned}
$$

Given this treatment, neither believe nor know can directly take a polar interrogative like whether Mary smokes as its complement, since the latter does not denote a proposition. Mayr (2017) assumes that this type clash can be resolved by letting the polar interrogative take sentential scope, leaving behind a trace of type $\langle s, t\rangle$. Thus, the logical form of such a construction is as follows:

$$
\text { [whether Mary smokes] } \lambda p[\text { John believes } p \text { ] }
$$

In order to determine what the interpretation of this logical form is, we need to specify how the excluded-middle presupposition projects out of the scope of an existential quantifier. Mayr (2017) does not specify this, but writes that the following interpretation is obtained:

$$
\lambda w_{s} \cdot \exists p \in Q^{\prime}: \underline{\operatorname{DOX}_{j}^{w} \subseteq p \vee \operatorname{DOX}_{j}^{w} \subseteq \bar{p}} . \operatorname{DOX}_{j}^{w} \subseteq p
$$

Note that it is not quite clear how this formula should be read. In particular, the underlined part cannot be read as usual, namely as restricting the domain of application of a certain function, because it does not come right after a lambda operator but rather appears in the scope of a quantifier which binds into it. Mayr (2017, p. 871) says the following about this:

"What is the presupposition of [(57)]? Taking the first of the propositions in $Q^{\prime}$ and setting it for $p$ in [(57)] gives the presupposition that John either believes that Mary smokes or that she does not smoke. Taking the second proposition in $Q^{\prime}$, however, yields exactly the same. As a consequence, the presupposition of $[(57)]$ is that John either believes that Mary smokes or that she does not smoke. Given the existential quantification in the assertive component of [(57)], the assertion is equivalent to the presupposition. This means that whenever [(57)]

\footnotetext{
${ }^{29}$ This interpretation actually differs slightly from the interpretation given in (17) of Mayr (2017). This is because, as confirmed by Mayr (p.c.), the latter contained a typo. The interpretation given here is the one that was intended.
} 
has a defined truth value, it is true. It is a tautology. Therefore [(56)] has a trivial literal meaning and is degraded..."

To determine whether this informal line of reasoning is tenable, we need a more general and precise specification of the assumed presupposition projection mechanism. Mayr (p.c.) has suggested that the desired results can be obtained by assuming a three-valued Strong Kleene logic, in which the truth value of $\exists x . \varphi(x)$ is defined just in case the truth value of $\varphi(x)$ is defined for at least one value of $x$, and $\exists x . \varphi(x)$ is true just in case $\varphi(x)$ is true for at least one value of $x$. Under this assumption, (57) is indeed true whenever it has a defined truth value. However, it remains to be seen whether a Strong Kleene logic is compatible with all the other parts of Mayr's proposal (concerning be certain, know, and other predicates).

Moreover, the account of Mayr (2017) is restricted to polar interrogative complements (the case of $w h$-interrogatives is explicitly left for future work), and it does not explicitly show that embedding polar interrogative clauses under believe and other neg-raising predicates always gives rise to logical analyticity (rather than just a tautology).

\section{A.1.2 Cohen $(2017 a, b)$}

Cohen $(2017 \mathrm{a}, \mathrm{b})$ has proposed two different ways of explaining the connection between anti-rogativity and neg-raising. We will not explicitly present these proposals here, for two reasons. First, they are cast in a different logical framework, which we would need to introduce in some detail before spelling out the proposals themselves. And second, the proposals are still rather preliminary at this point. One exists in the form of a 7-page handout, and the other in the form of a 5-page squib. Several important aspects have not been fully specified yet. For these reasons, we will restrict ourselves here to pointing out some challenges for the two proposals in their current form. A more comprehensive comparison must wait until the proposals have been worked out in more detail.

The proposal sketched in Cohen (2017a) wrongly predicts that under negation, negraising predicates do take interrogative complements. Moreover, it assumes that the EM presupposition of neg-raising predicates is pragmatic rather than semantic. As noted by Horn (1978), EM presuppositions are expected to arise much more widely under this assumption than they actually do. In particular, it becomes difficult, if not impossible, to account for the fact that predicates like believe trigger an EM presupposition while closely related predicates like be certain don't.

On the proposal sketched in Cohen (2017b) EM presuppositions are semantic in nature and neg-raising predicates are no longer predicted to license interrogative complements under negation. However, the account is, like that of Mayr (2017), restricted to polar interrogative complements. Moreover, it seems difficult to extend the account in a principled way to $w h$-interrogatives, because it relies on a non-compositional treatment of believing whether. This construction is, as a whole, viewed as a modal operator which comes with an EM presupposition. That is, the semantic contribution of believing whether is not derived from an independently motivated lexical entry for believe and an independently motivated treatment of interrogative complements. 
Finally, the proposal relies on a non-standard account of neg-raising, whose empirical coverage seems to be narrower than the account of neg-raising that we adopted, which is due to Gajewski (2007) building on much previous work. For instance, the account of Cohen (2017b) does not seem to account for the fact that negated neg-raising predicates license strong NPIs (e.g., Bill doesn't believe/*know that Mary has been back to England in years), a core empirical fact about neg-raising predicates (for discussion, see Gajewski 2007 and Križ 2015).

\section{A.2 Uegaki (2015b)}

We have shown that the selectional restrictions of some important classes of rogative and anti-rogative predicates can be derived from semantic assumptions about these predicates that can be independently motivated, and we argued that such an account is to be preferred over one that relies on a difference in semantic type between declarative and interrogative complements, at least as long as such a difference in type is not independently motivated.

Uegaki (2015b) assumes that declarative complements denote propositions, that interrogative complements denote sets of propositions, and that there is a type-shifting operation that transforms single propositions into sets of propositions if needed to avoid a type mismatch. This type-shifting operation, denoted Id, simply turns any proposition $p$ into the corresponding singleton set $\{p\}$. ${ }^{30}$

$$
\llbracket \mathrm{Id} \rrbracket^{w}=\lambda p \cdot\{p\}
$$

Thus, type-shifting is not needed when a responsive predicate like know takes an interrogative complement, as on the standard reductive approach (e.g., Heim 1994), but rather when such a predicate takes a declarative complement. For instance, John knows that Mary left is rendered as follows:

$$
\text { John knows [ld [that Mary left]] }
$$

In this setup, the selectional restrictions of anti-rogative predicates like believe can be seen as resulting from a type mismatch, under the assumption that such predicates require a single proposition as their input. On the other hand, the selectional restrictions of rogative predicates like wonder have to be given a different kind of explanation, because in terms of semantic type they do not differ from responsive predicates like know.

Uegaki provides such an explanation, as well as independent motivation for the assumed type distinction between anti-rogative predicates on the one hand and responsive and rogative predicates on the other. We will consider these aspects of Uegaki's proposal in Sects. A.2.1 and A.2.2, respectively, in each case drawing comparisons with our own approach.

\footnotetext{
30 In discussing Uegaki's proposal we adopt his convention to specify the denotation of each expression $\alpha$ at a specific world $w, \llbracket \alpha \rrbracket^{w}$, rather than the full meaning of the expression, $\llbracket \alpha \rrbracket$, which would be the function $\lambda w \cdot \llbracket \alpha \rrbracket^{w}$.
} 


\section{A.2.1 Rogative predicates}

Summary of Uegaki's account. The fact that wonder does not license declarative complements is accounted for by Uegaki (2015b, Sect. 2.3.3) in a way that is quite close in spirit to the account adopted in the present paper from Ciardelli and Roelofsen (2015), but different in implementation and empirical predictions. Uegaki proposes to decompose wonder into want to know and to derive the incompatibility with declarative complements from independently motivated assumptions about the lexical semantics of want. In particular, in line with earlier work on want, Uegaki (2015b, p.66) takes $x$ wants $p$ to presuppose (i) that $x$ believes that the presuppositions of $p$ are satisfied, and (ii) that $x$ does not believe that $p$ is true.

Uegaki's entry for want:

$\llbracket w a n t \rrbracket^{w}(p)(w)$ is defined only if:

(i) $\sigma_{x}^{w} \subseteq\left\{w^{\prime} \mid p\left(w^{\prime}\right)=1\right.$ or $\left.p\left(w^{\prime}\right)=0\right\} \quad$ ' $x$ believes presupposition of $p$ '

(ii) $\sigma_{x}^{w} \nsubseteq\left\{w^{\prime} \mid p\left(w^{\prime}\right)=1\right\} \quad$ ' $x$ does not believe that $p$ is true'

Now consider a case where wonder takes a declarative complement.

(61) *John wonders that Mary left.

If wonder is analyzed as want to know, then the truth value of (61) is only defined if (i) John believes that the presuppositions of John knows that Mary left are satisfied, i.e., he believes that Mary left, and (ii) John does not believe that John knows that Mary left is true. Assuming that $x$ believes $p$ generally entails $x$ believes that $x$ knows $p$, these two conditions are contradictory. Thus, it is predicted that the presuppositions of (61) can never be satisfied. This explains the fact that wonder does not license declarative complements, and Uegaki suggests that the account can be extended to other rogative predicates as well, assuming that all these predicates have want to know as a core component.

Problems and comparison. We see two problems for this proposal, one concerning the treatment of wonder itself, and one concerning the extension to other rogative predicates. Let us first consider the predictions of the account for a case where wonder takes an interrogative complement:

John wonders whether Mary left.

It is predicted that this sentence presupposes that John does not believe that John knows whether Mary left is true. Assuming that John is introspective, this is just to say that the sentence presupposes that John doesn't know whether Mary left. Since presuppositions under want project to the belief state of the subject (Karttunen 1973, 1974), it is therefore also predicted that (63) presupposes that John believes Mary doesn't know where he is.

John wants Mary to wonder where he is. 
This is a problematic prediction, because (63) can very well be true in a situation in which John believes that Mary already knows where he is. We take this to show that the 'ignorance component' of wonder is an entailment rather than a presupposition, and this is indeed how it is modeled on our account. As a result, we do not predict that (63) implies that John believes Mary is ignorant as to where he is.

Now let us turn to the possibility of extending Uegaki's account of wonder to other rogative predicates. It is indeed natural to assume that investigate and be curious are, just like wonder, very close in meaning to want to know. However, we do not think that this assumption is justifiable for predicates of dependency. It is clear that a sentence like (64) does not make reference to any agent's knowledge or desires, and can therefore not be paraphrased in terms of want to know.

Whether the light is on depends on whether the switch is up.

Thus, we think that the present proposal improves on Uegaki's account both in its treatment of wonder and in covering a broader range of predicates.

\section{A.2.2 Anti-rogative predicates}

Summary of Uegaki's account. As mentioned above, Uegaki assumes that antirogative predicates like believe require a single proposition as their input, while responsive and rogative predicates require sets of propositions. Moreover, he assumes that a declarative complement denotes a single proposition, while an interrogative complement denotes a set of propositions. This immediately accounts for the fact that anti-rogative predicates cannot take interrogative complements. Further assuming that a single proposition can be transformed into a set of propositions using the type-shifter Id, it is also predicted that responsive predicates can take both declarative and interrogative complements.

Uegaki motivates the assumption that anti-rogative predicates like believe and responsive predicates like know require different types of input by highlighting a contrast that arises when these two types of predicates are combined with so-called 'content DPs', like the rumor that Mary left. The contrast, first noted by Vendler (1972) and also discussed by Ginzburg (1995), King (2002), and Moltmann (2013), is illustrated in (65).

a. John believes the rumor that Mary left.

$\therefore$ John believes that Mary left.

b. John knows the rumor that Mary left.

$\%$ John knows that Mary left.

In general, $x$ believes the rumor that $p$ entails $x$ believes that $p$, whereas $x$ knows the rumor that $p$ does not entail $x$ knows that $p$, and the same is true if rumor is replaced by story, claim, hypothesis, et cetera.

Now, Uegaki claims that all anti-rogative predicates behave just like believe in this respect, while all responsive predicates behave just like know. He then provides an account of the contrast in (65) which relies on the assumption that believe requires a single proposition as its input, while know requires a set of propositions. Thus, 
to the extent that the account makes correct predictions for other anti-rogative and responsive predicates as well, it indeed provides independent motivation for the type distinction that Uegaki assumes to account for the selectional restrictions of antirogative predicates.

Problem and comparison. The problem for this approach is that there are counterexamples to the claim that all anti-rogative predicates behave like believe when combined with content DPs, and that all responsive predicates behave like know in this respect. First, there are anti-rogative predicates, such as think and want, which, unlike believe, cannot be combined with content DPs at all. ${ }^{31}$

*John thinks/wants/feels/supposes the rumor that Mary left.

While this does not directly counter Uegaki's account of the fact that believe is antirogative, it does show that the scope of the account is restricted; it certainly does not cover the full range of anti-rogative predicates.

A more drastic problem is that there are also responsive predicates that do not behave like know when combined with content DPs. Such predicates include hear and prove, as illustrated in (67)-(68). ${ }^{32}$

John heard the rumor that Mary left.

$\therefore$ John heard that Mary left.

John proved the hypothesis that every positive integer has a unique prime factorization.

$\therefore$ John proved that every positive integer has a unique prime factorization.

On Uegaki's account these predicates are thus predicted to be anti-rogative, just like believe, contrary to fact. This means that the independent motivation that Uegaki provides for his account of the selectional restrictions of anti-rogative predicates in terms of a type mismatch collapses. As a result, the account loses its explanatory force.

\footnotetext{
31 As an anonymous reviewer points out, these predicates can be combined with DPs like something and several things, expressions that Moltmann (2013) calls special quantifiers. With these expressions the relevant entailment is licensed:
}

(i) John thinks something-namely that Mary left.

$\therefore$ John thinks that Mary left.

However, as discussed above, we are interested here in the contrast between predicates like know and predicates like believe that arises with content nouns like rumor. With special quantifiers, on the other hand, there is no contrast between know and believe: as shown in (ii), if know combines with something the relevant inference is licensed (while with rumor it wouldn't be licensed). Hence, special quantifiers are not part of Uegaki's generalization, and therefore not a counterexample to our criticism.

(ii) John knows something — namely that Mary left.

$\therefore$ John knows that Mary left.

32 Uegaki (2015b, pp. 49,61) remarks that certain responsive predicates allow for a so-called entity-relating reading (such as the acquaintance reading of know), and that his theory leaves open the possibility that under this reading, these predicates do license inferences like those in (67)-(68). However, to the extent that such readings exist for hear and prove, they don't seem to be necessary for the inferences in (67)-(68) to go through. 
In comparison, we have shown that the selectional restrictions of two important classes of anti-rogative predicates can be derived from independently motivated semantic assumptions about these predicates, without the need to assume a mismatch in semantic type.

\section{Appendix B. Support-conditional lexical entries}

In the main text, we have given truth-conditional lexical entries for a number of predicates. For instance, according to our entry for be certain, repeated in (69) below, the predicate denotes a function which takes a complement meaning $P$ and an individual $x$ as its input, and delivers a truth value, either 1 or 0 , as its output, depending on the world of evaluation $w$.

$$
\text { «be certain } \rrbracket^{w}=\lambda P_{T} \cdot \lambda x . \operatorname{DOx}_{x}^{w} \in P
$$

Another, equivalent formulation of the entry is given in (70) below. This formulation makes clear that, when given a complement meaning $P$ and an individual $x$ as its input, the predicate yields a function from possible worlds to truth values. This kind of function can be identified with a set of possible worlds, namely those that are mapped to 1 . Such a set of worlds, a proposition, is taken to encode the meaning of a sentence in standard possible worlds semantics.

$$
\llbracket \text { be certain } \rrbracket=\lambda P_{T} \cdot \lambda x \cdot \lambda w \cdot \operatorname{DOx}_{x}^{w} \in P
$$

In inquisitive semantics, however, as discussed in Sect. 2, the meaning of a sentence is not a single proposition, but rather a set of propositions (non-empty and downward closed), encoding both the informative and the inquisitive content of the sentence. Thus, in inquisitive semantics, predicates like be certain should, when given a complement meaning $P$ and an individual $x$ as their input, not yield a set of worlds as their output, but rather a set of propositions-or equivalently, a function mapping every proposition $p$ either to 1 or to 0 . Schematically, the entries for such predicates should therefore be of the following form:

$$
\llbracket \cdot \rrbracket=\lambda P_{T} \cdot \lambda x \cdot \lambda p_{\langle s, t\rangle} \cdots
$$

In this way, a complete sentence like Bill is certain that Ann left is associated with a set of propositions, as desired. Each of these propositions is said to support the sentence. Thus, lexical entries that fit the scheme in (71) are called support-conditional, rather than truth-conditional, entries.

Now, what are the support-conditional entries of the predicates that we have discussed? We propose that they can be derived from their truth-conditional entries in a straightforward way. Namely, we assume that for every predicate $V$ under consideration, $\llbracket V \rrbracket(P)(x)(p)$ is defined just in case $\llbracket V \rrbracket(P)(x)(w)$ is defined for all $w \in p$, and $\llbracket V \rrbracket(P)(x)(p)=1$ just in case $\llbracket V \rrbracket(P)(x)(w)=1$ for all $w \in p$. Concretely, this yields the following entries:

$$
\llbracket \text { be certain } \rrbracket=\lambda P_{T} \cdot \lambda x \cdot \lambda p . \forall w \in p . \operatorname{DOX}_{x}^{w} \in P
$$




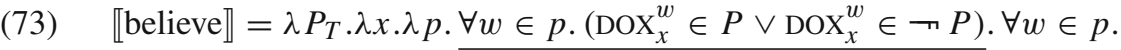
$\operatorname{DOX}_{x}^{w} \in P$

$$
\begin{aligned}
& \text { 匹be true }=\lambda P_{T} \cdot \lambda p . \forall w \in p . \mathrm{TRUE}^{w} \in P \\
& \text { 【be false } \rrbracket=\lambda P_{T} \cdot \lambda p . \forall w \in p . \operatorname{TRUE}^{w} \notin P \\
& \llbracket \text { wonder } \rrbracket=\lambda P_{T} . \lambda x . \lambda p . \forall w \in p .\left(\operatorname{DOx}_{x}^{w} \notin P \wedge \mathrm{INQ}_{x}^{w} \subseteq P\right) \\
& \llbracket \text { depend on } \rrbracket=\lambda P_{T}^{\prime} . \lambda P_{T} \cdot \lambda p . \forall w \in p . \exists f \in \operatorname{alt}(P)^{\operatorname{alt}\left(P^{\prime}\right)} \text { such that: }
\end{aligned}
$$

To briefly illustrate what these entries deliver, consider the following sentence:

John wonders whether Mary called.

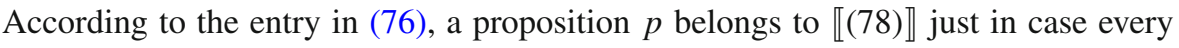
world $w \in p$ is one in which (i) John isn't certain yet whether Mary called, but (ii) every extension of his current doxastic state in which the issues that he entertains are

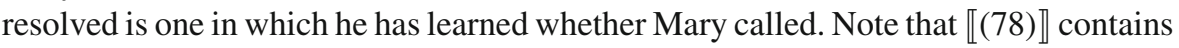
a single maximal element, i.e., a single alternative, which is the set of all worlds in which conditions (i) and (ii) above are satisfied. Thus, it is correctly predicted that (78) is not inquisitive, and that the sentence is true in $w$, i.e., $w \in$ info( $(78) \rrbracket)$, exactly when it is true according to our truth-conditional entry for wonder in (37).

More generally, our support-conditional entries predict for any predicate $V$ under consideration, and any declarative sentence $\varphi$ in which $V$ takes a clausal complement and an individual subject (or two clausal complements in the case of depend on), that (i) $\varphi$ is non-inquisitive, and (ii) $\varphi$ is true in a world $w$, i.e., $w \in \operatorname{info}(\varphi)$, just in case it is true in $w$ according to our truth-conditional entries.

Indeed, because of this tight connection between the support and truth conditions of sentences involving the predicates in question, we felt justified in concentrating only on the latter in the main text of the paper. For more details concerning type-theoretic inquisitive semantics, we refer to Ciardelli et al. (2017).

\section{Appendix C. Extending the account to presuppositional questions}

In this appendix we demonstrate how our account can be extended to presuppositional questions. Such questions are problematic for our account in its current form because it derives the selectional restrictions of anti-rogatives from the fact that the meaning of an interrogative complement always covers the entire logical space $W$. Presuppositional questions, however, do not cover $W$, but only a subset of $W$.

\section{C.1 Presuppositional questions}

Let us consider the example of a polar question containing the presupposition trigger stop: 
Did John stop smoking?

As before, we model presuppositions via definedness restrictions: e.g., [Did John stop smoking? $\rrbracket(p)$ is defined only if John used to smoke in all worlds $w \in p$ :

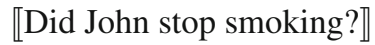

$$
=\lambda p \cdot \begin{cases}\forall w \in p: S(w)(j) \vee \forall w \in p: \neg S(w)(j) & \text { if } \forall w \in p: U(w)(j) \\ \text { undefined } & \text { otherwise }\end{cases}
$$

In line with this, we define the presupposition $\pi(P)$ of a sentence meaning $P$ as the set of all those propositions $p$ for which $P(p)$ is defined.

Definition 1 (Presupposition)

The presupposition $\pi(P)$ of a sentence meaning $P$ is $\pi(P)=\{p \mid P(p)$ is defined $\}$.

\section{C.2 Presupposition projection}

Negation. It is well known that presuppositions project through negation. We modify the definition of the inquisitive negation operator to model this fact.

$$
\boldsymbol{\tau}=\lambda P . \lambda p \cdot \begin{cases}\forall q \in P: p \cap q=\emptyset & \text { if } P(p) \text { is defined } \\ \text { undefined } & \text { otherwise }\end{cases}
$$

As before, when $\rightarrow$ is applied to a sentence meaning $P$, it again yields a sentence meaning, i.e., a set of propositions. Now, however, $\rightarrow P(p)$ is only defined if $P(p)$ is. As a consequence, for any sentence meaning $P$, it holds that $\pi(P)=\pi(\neg P)$.

Embedding predicates. Next, we turn to the embedding predicate believe. As observed by Karttunen (1973, 1974), a sentence like (82) presupposes not that John used to smoke, but that Mary believes that he used to smoke. That is, believe and other non-factive attitude predicates project the presupposition of their complement clause by attributing it to the attitude holder as a belief.

(82) Mary believes that John stopped smoking.

Presupposition: Mary believes that John used to smoke.

The support-conditional version of the existing lexical entry for believe is repeated in (83) (see Appendix B). Recall that the definedness restriction of this entry serves to model the excluded-middle (EM) presupposition. In what follows, we will refer to the disjunction modelling the EM presupposition $\left(\forall w \in p: \operatorname{DOX}_{x}^{w} \in P\right.$ or $\forall w \in p$ : $\operatorname{DOX}_{x}^{w} \in \rightarrow P$ ) as the EM condition.

$$
\begin{aligned}
& \text { 【believe』 } \\
& =\lambda P . \lambda x . \lambda p \cdot\left\{\begin{aligned}
& \forall w \in p: \operatorname{DOx}_{x}^{w} \in P \text { if } \forall w \in p: \operatorname{DOx}_{x}^{w} \in P \text { or } \\
& \forall w \in p: \operatorname{DOx}_{x}^{w} \in \rightarrow P \\
& \text { undefined } \text { otherwise }
\end{aligned}\right.
\end{aligned}
$$


In order to also model the presupposition projection behavior of believe, we may add another condition to the existing definedness restriction. What we require for

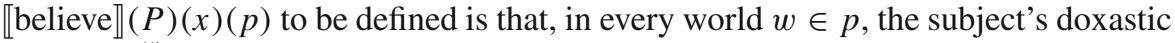
state, $\operatorname{DOX}_{x}^{w}$, satisfies the presuppositions of the complement meaning $P$. That is, for every $w \in p, P\left(\operatorname{DOX}_{x}^{w}\right)$ should be defined. A lexical entry for believe including this additional condition is given in (84).

$$
=\lambda P . \lambda x \cdot \lambda p \cdot \begin{cases}\forall w \in p: \operatorname{DOx}_{x}^{w} \in P & \text { if }\left(\forall w \in p: \operatorname{DOx}_{x}^{w} \in P \text { or } \forall w \in p:\right. \\ & \left.\operatorname{DOx}_{x}^{w} \in \rightarrow P\right) \\ & \text { and } \forall w \in \boldsymbol{p}: \mathbf{D O X}_{x}^{w} \in P \text { is defined } \\ \text { undefined } & \text { otherwise }\end{cases}
$$

However, because presuppositions project through negation, we find that whenever $p$ satisfies the EM condition, it is of course also defined for all $w \in p$ whether $\operatorname{DOX}_{x}^{w} \in P$. This means that we may also just omit the additional definedness condition and stick with the entry in (83).

\section{C.3 Relativizing non-informativity}

Recall that we assume that interrogative complements are never informative. We had taken a sentence $\varphi$ to be non-informative iff its informative content is trivial-by which we meant trivial w.r.t. the logical space $W$. That is, we called $\varphi$ non-informative iff $\operatorname{info}(\varphi)=W$. Now that we are also considering presuppositional questions, it is natural to relativize the definition of non-informativity to the presuppositional content of a sentence. We say that a sentence $\varphi$ with presupposition $\pi(\llbracket \varphi \rrbracket)$ is non-informative w.r.t. its presupposition iff info $(\varphi)=\bigcup \pi(\llbracket \varphi \rrbracket)$. This is the case iff the alternatives in $\llbracket \varphi \rrbracket$ together cover $\bigcup \pi(\llbracket \varphi \rrbracket)$. Intuitively, we can think of this along the following lines. Suppose that info $(\varphi)=\bigcup \pi(\llbracket \varphi \rrbracket)$ and consider the doxastic state of someone who hears $\varphi$. Then, whenever this doxastic state is one that satisfies the presupposition of $\varphi$, i.e., an element of $\pi(\llbracket \varphi \rrbracket)$, it will also already contain all the information encoded by $\operatorname{info}(\varphi)$, i.e., $\varphi$ will not add any information to the given doxastic state.

Using this relativized notion, we now assume that interrogative complements are always non-informative w.r.t. their presupposition. In case an interrogative does not carry a presupposition, this simply boils down to normal non-informativity.

At this point, we can already see from the definition of inquisitive negation in (81) that, just as before, the inquisitive negation of an interrogative complement meaning $P$ with presupposition $\pi(P)$ is always $\rightarrow P=\{\emptyset\}$. This is because there can be no nonempty proposition $p \in \pi(P)$ such that $p$ is inconsistent with every $q \in P$.

Also as before, this means that if believe takes an interrogative complement, the second disjunct of the EM condition can only be true if $\operatorname{DOX}_{x}^{w}=\emptyset$. It follows that the second disjunct can only be true if the first disjunct is true as well, since $\emptyset$ is contained in any complement meaning $P$. In other words, the second disjunct in the EM condition is redundant. Thus, if believe takes an interrogative complement, its lexical entry reduces to (85). 


$$
\begin{aligned}
& \text { 【believe』 } \\
& =\lambda P . \lambda x . \lambda p \cdot \begin{cases}\forall w \in p: \operatorname{DOX}_{x}^{w} \in P & \text { if } \forall w \in p: \operatorname{DOX}_{x}^{w} \in P \\
\text { undefined } & \text { otherwise }\end{cases}
\end{aligned}
$$

Note that the definedness condition in (85) entails the support condition. In other words, when believe combines with an interrogative complement, its support condition is trivial relative to its presupposition. We will now again show that this triviality is a case of L-analyticity.

\section{C.4 L-analyticity}

It is straightforward to translate the notion of L-analyticity into our support-conditional framework:

Assumption 3 (L-analyticity, support-based version) A sentence $S$ with logical skeleton $\chi$ is L-analytical just in case either (i) or (ii) holds.

(i) For all interpretations, if it is defined whether a proposition $p$ supports $\chi$, then $p$ supports $\chi$.

(ii) For all interpretations, if it is defined whether a proposition $p$ supports $\chi$, then $p$ does not support $\chi$.

We now show that the meaning of (86) still comes out as L-analytical on the presuppositional account.

(86) *Mary believes whether John stopped smoking.

We again start by constructing the logical skeleton (LS). As before, we assume that believe decomposes at LF into $\mathcal{M}_{\text {believe }}$ and $R_{\mathrm{EM}}$. The lexical entries of these items need to be modified slightly to fit the support-conditional setting.

$$
\begin{aligned}
& \llbracket \mathcal{M}_{\text {believe }}(w)(x) \rrbracket=\operatorname{DOX}_{x}^{w} \\
& \llbracket R_{\mathrm{EM} \rrbracket} \\
= & \lambda \mathcal{M}_{\langle s,\langle\mathrm{e}, \mathrm{st}\rangle\rangle \cdot \lambda P . \lambda x \cdot \lambda p .} \\
& \begin{cases}\forall w \in p: \mathcal{M}(w)(x) \in P & \text { if }(\forall w \in p: \mathcal{M}(w)(x) \in P \text { or } \\
& \forall w \in p: \mathcal{M}(w)(x) \in \rightarrow P) \\
\text { undefined } & \text { otherwise }\end{cases}
\end{aligned}
$$

The LS for (86) is given in (89).

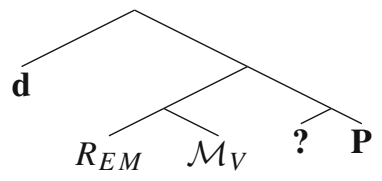

Now, let $p$ be a proposition. We want to determine whether $p \in \llbracket(89) \rrbracket$. Whether this is the case, though, is only defined if $p$ supports the presupposition $\pi(\llbracket(89) \rrbracket)$. 
This means it has to hold that either (a) $\llbracket \mathcal{M} \rrbracket(w)(\llbracket \mathbf{d} \rrbracket) \in \llbracket \mathbf{P} \rrbracket$ for all $w \in p$, or (b) $\llbracket \mathcal{M} \rrbracket(w)(\llbracket \mathbf{d} \rrbracket) \in \llbracket \neg \mathbf{?} \mathbf{P} \rrbracket$ for all $w \in p$. We already know that, no matter what $\mathbf{P}$ is, the set of propositions in $\llbracket \mathbf{P P} \rrbracket$ covers the presupposition of $\mathbf{P P}, \pi(\llbracket \mathbf{P} \rrbracket)$. That is, info $(\mathbf{P})=\bigcup \pi(\llbracket ? \mathbf{P} \rrbracket)$. Hence, we also know that $\llbracket \rightarrow \mathbf{?} \rrbracket=\{\emptyset\}$.

The second disjunct in the EM condition, (b), can thus only be true if $\llbracket \mathcal{M} \rrbracket(w)(\llbracket \mathbf{d} \rrbracket)=$ $\emptyset$ for all $w \in p$. But if this holds, then the first disjunct is also true, since $\llbracket \mathbf{P P} \rrbracket$ always contains $\emptyset$. This means that whenever the second disjunct holds, the first one holds as well, or, in other words, whenever the EM condition holds, the first disjunct is true.

Now, let's assume that the $p$ we were considering indeed supports the presupposition $\pi(\llbracket(89) \rrbracket)$. Then we know that the first disjunct of the EM condition holds. But note that this disjunct is identical to the support condition for $p$, namely $\llbracket \mathcal{M} \rrbracket(w)(\llbracket \mathbf{d} \rrbracket) \in \llbracket \mathbf{P} \mathbb{P} \rrbracket$ for all $w \in p$. This in turn means that, for all interpretations in which it is defined whether $p \in \llbracket(89) \rrbracket$, it is indeed the case that $p \in \llbracket(89) \rrbracket$. Hence, (89) comes out as L-analytical.

\section{References}

Abels, K. 2004. Why surprise-predicates do not embed polar interrogatives. Linguistische Arbeitsberichte 81: 203-222.

Abrusán, M. 2014. Weak island semantics. Oxford: Oxford University Press.

Abusch, D. 2002. Lexical alternatives as a source of pragmatic presuppositions. In Proceedings of SALT 12, ed. B. Jackson, 1-19. Ithaca, NY: CLC Publications.

Abusch, D. 2010. Presupposition triggering from alternatives. Journal of Semantics 27: 37-80.

AnderBois, S. 2012. Focus and uninformativity in Yukatek Maya questions. Natural Language Semantics 20: 349-390.

AnderBois, S. 2014. The semantics of sluicing: Beyond truth conditions. Language 90 (4): 887-926.

Bartsch, R. 1973. "Negative transportation" gibt es nicht. Linguistische Berichte 27 (7): 1-7.

Barwise, J., and R. Cooper. 1981. Generalized quantifiers and natural language. Linguistics and Philosophy 4 (2): 159-219.

Bošković, Ž., and J. Gajewski. 2011. Semantic correlates of the NP/DP parameter. In Proceedings of NELS 39, ed. S. Lima, K. Mullin, and B. Smith, 121-134. Amherst, MA: GLSA.

Ciardelli, I. 2016. Questions in logic. Ph.D. dissertation, ILLC, University of Amsterdam.

Ciardelli, I., J. Groenendijk, and F. Roelofsen. 2012. Inquisitive semantics. NASSLLI lecture notes. http:// projects.illc.uva.nl/inquisitivesemantics.

Ciardelli, I., J. Groenendijk, and F. Roelofsen. 2013. Inquisitive semantics: A new notion of meaning. Language and Linguistics Compass 7 (9): 459-476.

Ciardelli, I., J. Groenendijk, and F. Roelofsen. 2015. Inquisitive semantics. ESSLLI lecture notes. http:// projects.illc.uva.nl/inquisitivesemantics.

Ciardelli, I., J. Groenendijk, and F. Roelofsen. 2018. Inquisitive semantics. Oxford: Oxford University Press.

Ciardelli, I., and F. Roelofsen. 2015. Inquisitive dynamic epistemic logic. Synthese 192 (6): 1643-1687.

Ciardelli, I., F. Roelofsen, and N. Theiler. 2017. Composing alternatives. Linguistics and Philosophy 40 (1): $1-36$.

Cohen, M. 2017a. Neg-raising and question embedding. Talk presented at the UCSC-Stanford Workshop on Sentence Types, Jan. 29, 2017.

Cohen, M. 2017b. A note on belief, question embedding and neg-raising. In Proceedings of LORI 2017, ed. A. Baltag, J. Seligman, and T. Yamada, 648-652. Berlin: Springer.

d'Avis, F.J. 2002. On the interpretation of wh-clauses in exclamative environments. Theoretical Linguistics 28 (1): 5-31.

Dayal, V. 1996. Locality in wh-quantification: Questions and relative clauses in Hindi. Dordrecht: Kluwer Academic Publishers.

Egré, P. 2008. Question-embedding and factivity. Grazer Philosophische Studien 77: 85-125. Special issue on Knowledge and Questions, ed. F. Lihoreau. 
Elliott, P.D., N. Klinedinst, Y. Sudo, and W. Uegaki. 2017. Predicates of relevance and theories of question embedding. Journal of Semantics 34 (3): 547-554.

Gajewski, J. 2002. L-analyticity and natural language. Manuscript, MIT.

Gajewski, J.R. 2005. Neg-raising: Polarity and presupposition. Ph.D. dissertation, MIT.

Gajewski, J.R. 2007. Neg-raising and polarity. Linguistics and Philosophy 30 (3): 289-328.

Gajewski, J.R. 2009. L-triviality and grammar. Handout of a talk given at the UConn Logic Colloquium, Feb. 27, 2009.

George, B. 2011. Question embedding and the semantics of answers. Ph.D. dissertation, University of California, Los Angeles.

Ginzburg, J. 1995. Resolving questions, I \& II. Linguistics and Philosophy 18(5/6): 459-527, 567-609.

Guerzoni, E. 2007. Weak exhaustivity and whether: A pragmatic approach. In Proceedings of SALT 17, ed. T. Friedman and M. Gibson, 112-129. Ithaca, NY: CLC Publications.

Heim, I. 1994. Interrogative semantics and Karttunen's semantics for know. In The Proceedings of IATL 9 , ed. R. Buchalla and A. Mittwoch. Academon: Jerusalem.

Horn, L. 1978. Remark on neg-raising. Syntax and Semantics 9: 129-220.

Horn, L.R. 1989. A natural history of negation. Chicago: The University of Chicago Press.

Karttunen, L. 1973. The last word. Manuscript. Austin: University of Texas.

Karttunen, L. 1974. Presupposition and linguistic context. Theoretical Linguistics 1 (1-3): 181-194.

Karttunen, L. 1977. Syntax and semantics of questions. Linguistics and Philosophy 1: 3-44.

Katzir, R., and R. Singh. 2013. Hurford disjunctions: Embedded exhaustification and structural economy. In Sinn und Bedeutung 18, ed. U. Etxeberria, A. Fălăuş, A. Irurtzun, and B. Leferman, 201-216. Bilbao: University of the Basque Country.

King, J.C. 2002. Designating propositions. The Philosophical Review 111 (3): 341-371.

Kratzer, A., and J. Shimoyama. 2002. Indeterminate pronouns: The view from Japanese. In The third Tokyo conference on psycholinguistics, ed. Y. Otsu, 1-25. Tokyo: Hituzi.

Križ, M. 2015. Aspects of homogeneity in the semantics of natural language. Ph.D. dissertation, University of Vienna.

Lahiri, U. 2002. Questions and answers in embedded contexts. Oxford: Oxford University Press.

Mayr, C. 2017. Predicting polar question embedding. In Proceedings of Sinn und Bedeutung 21, ed. R. Truswell, 863-880. Edinburgh: University of Edinburgh.

Mayr, C., and J. Romoli. 2016. A puzzle for theories of redundancy: Exhaustification, incrementality, and the notion of local context. Semantics and Pragmatics 9 (7): 1-48.

Moltmann, F. 2013. Abstract objects and the semantics of natural language. Oxford: Oxford University Press.

Nicolae, A.C. 2013. Any questions? Polarity as a window into the structure of questions. Ph.D. dissertation, Harvard.

Roberts, T. 2018. Responsive predicates are question-embedding: Evidence from Estonian. In Proceedings of $S u B$ 22, eds. U. Sauerland and S. Solt, 271-288.

Roelofsen, F. 2013a. Algebraic foundations for the semantic treatment of inquisitive content. Synthese 190 (1): 79-102.

Roelofsen, F. 2013b. An inquisitive perspective on meaning: the case of disjunction. http://projects.illc.uva. nl/inquisitivesemantics. Talk presented at Stanford Linguistics Colloquium, February 2013.

Roelofsen, F. 2015. The semantics of declarative and interrogative lists. Manuscript, ILLC, University of Amsterdam.

Roelofsen, F. 2017. Surprise for Lauri Karttunen. Manuscript, ILLC, to appear in Lauri Karttunen Festschrift, ed. Cleo Condoravdi.

Roelofsen, F., M. Herbstritt, and M. Aloni. 2019. The *whether puzzle. In Questions in discourse, ed. K. von Heusinger, E. Onea, and M. Zimmermann. Leiden: Brill.

Romero, M. 2015. Surprise-predicates, strong exhaustivity and alternative questions. In Proceedings of SALT 25, ed. S. D’Antonio, M. Moroney, and C.R. Little, 225-245. Ithaca, NY: CLC Publications.

Romoli, J. 2013. A scalar implicature-based approach to neg-raising. Linguistics and Philosophy 36 (4): 291-353.

Sæbø, K.J. 2007. A whether forecast. In Logic, language, and computation, ed. B. ten Cate and H. Zeevat, 189-199. Berlin: Springer.

Schlenker, P. 2009. Local contexts. Semantics and Pragmatics 2 (3): 1-78.

Spector, B., and P. Egré. 2015. A uniform semantics for embedded interrogatives: An answer, not necessarily the answer. Synthese 192 (6): 1729-1784. 
Steinert-Threlkeld, S. 2019. An explanation of the veridical uniformity universal. Journal of Semantics (forthcoming).

Steinert-Threlkeld, S. and J. Szymanik 2019. Learnability and semantic universals. Manuscript, ILLC, to appear in Semantics and Pragmatics.

Theiler, N., F. Roelofsen, and M. Aloni. 2018. A uniform semantics for declarative and interrogative complements. Journal of Semantics 35 (3): 409-466.

Uegaki, W. 2015a. Content nouns and the semantics of question-embedding. Journal of Semantics 33 (4): 623-660.

Uegaki, W., 2015b. Interpreting questions under attitudes. Ph.D. dissertation, MIT.

Uegaki, W., and F. Roelofsen. 2018. Do modals take propositions or sets of propositions? Evidence from Japanese darou. In Proceedings of SALT 28, ed. S. Maspong, B. Stefánsdóttir, K. Blake, and F. Davis, 809-829. Washington, DC: LSA.

Uegaki, W., and Y. Sudo. 2017. The anti-rogativity of non-veridical preferential predicates. In Proceedings of the 21st Amsterdam Colloquium, ed. A. Cremers et al., 492-501. Amsterdam: ILLC.

van Gessel, T., A. Cremers, and F. Roelofsen. 2018. Polarity sensitivity of question embedding: Experimental evidence. In Proceedings of SALT 28, ed. M. Maspong, B. Stefánsdóttir, K. Blake, and F. Davis, 217232. Washington, DC: LSA.

Vendler, Z. 1972. Res cogitans: An essay in rational psychology. Ithaca, NY: Cornell University Press.

White, A.S., and K. Rawlins. 2016. A computational model of S-selection. In Proceedings of SALT 26, ed. M. Moroney, C.R. Little, J. Collard, and D. Burgdorf, 641-663. Washington, DC: LSA.

Zuber, R. 1982. Semantic restrictions on certain complementizers. In Proceedings of the 13th international congress of linguists, Tokyo, ed. S. Hattori and K. Inove, 434-436. Tokyo: Proceedings Publishing Committee.

Publisher's Note Springer Nature remains neutral with regard to jurisdictional claims in published maps and institutional affiliations. 